\title{
1 Insights into the drivers of radiating diversification in biodiversity 2 hotspots using Saussurea (Asteraceae) as a case
}

4 Xu Zhang ${ }^{1,2,3 *}$, Jacob B. Landis ${ }^{4,5 *}$, Yanxia Sun ${ }^{1,2}$, Huajie Zhang ${ }^{1}$, Tao Feng ${ }^{1}$, Nan Lin ${ }^{1}$, Bashir B.

5 Tiamiyu $^{1}$, Xianhan Huang ${ }^{6}$, Tao Deng ${ }^{6}$, Hengchang Wang ${ }^{1,2}$, Hang Sun ${ }^{6}$

$6 \quad{ }^{1}$ CAS Key Laboratory of Plant Germplasm Enhancement and Specialty Agriculture, Wuhan Botanical

7 Garden, Chinese Academy of Sciences, Wuhan 430074, Hubei, China;

$8 \quad{ }^{2}$ Center of Conservation Biology, Core Botanical Gardens, Chinese Academy of Sciences, Wuhan

9 430074, Hubei, China;

$10{ }^{3}$ University of Chinese Academy of Sciences, Beijing, 100049 China;

$11{ }^{4}$ School of Integrative Plant Science, Section of Plant Biology and the L.H. Bailey Hortorium, Cornell

12 University, Ithaca, NY 14850, USA;

$13{ }^{5}$ BTI Computational Biology Center, Boyce Thompson Institute, Ithaca, NY 14853, USA;

$14{ }^{6}$ Key Laboratory for Plant Diversity and Biogeography of East Asia, Kunming Institute of Botany,

15 Chinese Academy of Sciences, Kunming, Yunnan, 650201 China

$16 *$ These authors contributed equally to this work.

18 Authors for correspondence:

19 Tao Deng

20 Email: dengtao@mail.kib.ac.cn

22 Hengchang Wang

23 Email: hcwang@wbgcas.cn

24

25 Hang Sun

26 Email: sunhang@mail.kib.ac.cn 


\section{$30 \quad$ Abstract}

31 - The Qinghai-Tibet Plateau (QTP) encompasses areas with a remarkably high degree

32 of biodiversity, harboring exceptional species-rich radiations. How these radiations

33 formed by interacting with geology, climate and ecology remains seldom examined.

34 - We investigate the roles of abiotic (environmental) and biotic (species-intrinsic)

35 factors in driving radiating diversification of Saussurea (Asteraceae) by deploying a

36 number of time-dependent, paleoenvironment-dependent and trait-dependent models, as

37 well as ecological distribution data.

38 - We show that three main clades of Saussurea begin to diversify in the Miocene

39 almost simultaneously, with increasing diversification rates toward the present and

40 negative dependence to paleotemperature. Acceleration in diversification rates are

41 correlated with adaptive traits, as well climate lability, niche breadth and species range.

42 - We conclude that fluctuation of paleoclimate along with complex QTP environments

43 provided opportunities for increased diversification rates of Saussurea with diverse

44 adaptive traits, highlighting the importance of combinations of clade-specific traits and

45 ecological niches in driving rapid radiation.

46 Key words: radiating diversification, Saussurea, the Qinghai-Tibet Plateau, biodiversity

47 hotspots, adaptive traits, diversification rates, ecological niche. 


\section{Introduction:}

50 The diversification pattern of species-rich rapid radiations reflects the evolutionary

51 dynamics of biodiversity hotspots (Linder \& Verboom, 2015). Understanding how these

52 radiating lineages formed in response to historical process can advance our knowledge of

53 adaptive evolution and enhance our ability to predict the threats to biodiversity posed by

54 global warming (Ding et al., 2020). Mountainous regions represent just one-eighth of

55 terrestrial land surface but are home to one-third of all species and exceptional species-

56 rich radiations (Antonelli, 2015; Schwery et al., 2015; Antonelli et al., 2018). Particularly

57 enigmatic is the Qinghai-Tibet Plateau (QTP) region, also known as the "Third Pole,"

58 characterized by a complex geographical history and encompassing areas of remarkably

59 high degree of biodiversity (Favre et al., 2015; Xing \& Ree, 2017; Chen et al., 2018;

60 Ding et al., 2020; Spicer et al., 2020). The QTP stands out as the earth's highest and

61 largest plateau, and includes the Himalaya and Hengduan Mountains which are listed as

62 two of the 36 hotspots of biodiversity in the world (Myers et al., 2000; Li et al., 2014;

63 Wen et al., 2014; Favre et al., 2015). The presence of steep environmental gradients in

64 temperature and precipitation create abundant micro-habitats providing a variety of

65 ecological niches essential for evolutionary radiations on the QTP (Mosbrugger et al.,

66 2018; Muellner-Riehl et al., 2019). While a plethora of studies have suggested that

67 diversification of plants on the QTP have evolved in association with plateau uplifting

68 processes (reviewed by Wen et al., 2014), how such high species diversity form in such a

69 short period of geologic time, and the interactions with geography, climate and ecology,

70 remain seldom examined.

71 Evolutionary and diversification patterns of plants are often correlated with

72 environmental abiotic forces, such as abrupt changes in climate or geological tectonic

73 events that drive speciation and extinction rates, and/or species-intrinsic/biotic factors, 
74 such as interactions among species and key innovation traits (Drummond et al., 2012;

75 Hughes \& Atchison, 2015; Condamine et al., 2018; Muellner-Riehl et al., 2019; Nürk et

76 al., 2019). There is a gap in our current understanding of radiating diversification drivers

77 in the flora of the QTP, with previous studies mostly providing only a temporal

78 (molecular dating) framework associating rapid radiations with the time span of plateau

79 uplifting (e.g. Wang et al., 2009; Zhang et al., 2014; Xu et al., 2019). Employing models

80 assuming continuous variation in diversification rates over time that depend on

81 paleoenvironmental variables is essential to precisely determine how diversification rates

82 are affected by abiotic environmental changes (Condamine et al., 2013; Sun et al., 2020).

83 In addition to abiotic factors, diversification shifts are often correlated with the evolution

84 of certain functional traits (Hughes \& Atchison, 2015). Examples include geophytism in

85 monocots leading to higher rates of diversification (Howard et al., 2020),

86 polyploidization promoting species diversification of Allium (Han et al., 2020), and

87 pollinator shifts, fruit types as well as elevational changes in the Andean bellflowers

88 (Lagomarsino et al., 2016). Furthermore, the inclusion of ecological niche data is also

89 crucial, because this reflects the interplay between historical processes and species

90 intrinsic factors (Lavergne et al., 2010; Folk et al., 2019; Muellner-Riehl et al., 2019).

91 Here, we address the knowledge gap of rapid diversification by examining the roles

92 of abiotic (environmental) and biotic (species-intrinsic) factors in driving radiating

93 diversification of the species-rich genus Saussurea DC. (Asteraceae). Saussurea is one of

94 the most diverse genera in Asteraceae, serving as an ideal study system for investigating

95 the evolutionary patterns of a rapid radiation. The genus comprises approximately 400

96 species that are distributed in Asia, Europe and North America, with the highest diversity

97 in the QTP (Wang et al., 2009; Shi \& Raab-Straube, 2011; Chen, 2015; Zhang, et al.,

98 2019a). Uncertainty in the number of species has largely been attributed to the complex

99 taxonomy of related QTP taxa (Chen \& Yuan, 2015), indicative of a recent radiation. 
100 Saussurea exhibits extraordinary morphological diversity. For example, the most

101 impressive species groups are the 'snowball plants' or 'snow rabbits', $S$. subg.

102 Eriocoryne, with a thick woolly indumentum (densely haired), and the so-called

103 'greenhouse plants' or 'snow lotuses', S. subg. Amphilaena, in which the synflorescence

104 is hidden by semi-transparent, white, yellowish or purple leafy bracts (Shi \& Raab-

105 Straube, 2011; Chen, 2015). Saussurea is present in virtually all possible habitats of the

106 QTP, including steppes, moist forests, cold and dry alpine meadows, and scree slopes

107 above 5,000 m, demonstrating a highly adaptive capability (Shi \& Raab-Straube, 2011).

108 Previous studies suggested that attractive morphological traits were the result of

109 convergent adaptation to diverse environments in the QTP (Kita et al., 2004; Wang et al.,

110 2009; Zhang, et al., 2019a), yet their contributions to the high-level diversity of

111 Saussurea are still elusive. While biogeographic analysis inferred that Saussurea arose

112 during the Miocene in the Hengduan Mountains (Xu et al., 2019), limited information

113 about macro-evolutionary patterns related to historical climate and geologic processes

114 were provided due to the lack of modeling diversification rates.

115 A robust phylogenetic framework is the basis for large-scale analyses of evolutionary

116 patterns (Koenen et al., 2020), yet previous studies mainly relied on fragment DNA

117 markers (e.g. Han et al., 2020; Howard et al., 2020; Sun et al., 2020), which have been

118 revealed to provide insufficient phylogenetic signals and always yield parallel

119 relationships for phylogenies of rapid radiations (Whitfield \& Lockhart, 2007; Wang et

120 al., 2009). In the present study, we reconstructed a robust time-calibrated phylogeny of

121 Saussurea using 226 complete plastomes to explore the role played by abiotic and biotic

122 factors in this rapidly radiating clade. If evolutionary dynamics are driven primarily by

123 abrupt abiotic perturbations, we would expect diversification rate shifts following major

124 climate changes that extirpated certain lineages while favoring the radiation of others. In

125 contrast, if biotic factors or interactions among species are the dominant drivers of 
126 evolution, we would expect diversification shifts to be correlated with the evolution of

127 functional traits and/or the colonization of new habitats (Condamine et al., 2018). While

128 in a joint-effect scenario, diversification rates may vary continuously through time and

129 paleoenvironments may shift with some clade-specific traits. We could hypothesize that

130 fluctuations of terrestrial and climatic systems provide vast ecological opportunities,

131 which are seized by lineages with ample adaptive traits and promote rapid radiating,

132 emphasizing the decisive role of morphological diversity/plasticity and ecological niche

133 availability. To test these hypotheses, we deployed a number of time-dependent,

134 paleoenvironment-dependent and trait-dependent models, as well as ecological

135 distribution data. Our study is designed to address the effects of paleoenvironmental and

136 biological drivers on radiating diversification in the biodiversity hotspots, while

137 providing a compelling example of the pivotal roles of morphological diversity and

138 ecological niche.

\section{Materials and Methods}

\section{Plastome Sampling, Sequencing and Assembly}

141 To build a dated phylogeny of the genus Saussurea, we newly sequenced plastomes for

14263 species and downloaded 163 additional plastomes from GenBank (accessed 29

143 November 2019); collectively these species included 199 taxa of Saussurea and 27

144 outgroup taxa. Collection details of the specimens were provided in Supporting

145 Information Table S1. Total genomic DNA of was extracted from silica-gel dried leaves

146 with a modified hexadecyltrimethylammonium bromide (CTAB) method (Yang et al.,

147 2014). Purified DNA was fragmented and used to construct short-insert (500 bp) libraries

148 per the manufacturer's instructions (Illumina). Libraries were quantified using an Agilent

1492100 Bioanalyzer (Agilent Technologies, Santa Clara, CA, USA), and were then

150 sequenced on an Illumina HiSeq 4000 platform at Novogene Co., Ltd. in Kunming, 
151 Yunnan, China. Raw reads were directly assembled with the organellar assembler

152 NOVOPLASTY v.2.7.2 (Dierckxsens et al., 2017), using a seed-and-extend algorithm

153 employing the plastome sequence of Saussurea japonica (Genbank accession:

154 MH926107.1) as the seed input, and all other parameters kept at default settings.

155 Assembled plastome sequences were initially annotated using Plastid Genome Annotator

156 (PGA) (Qu et al., 2019), and then manually checked in GENEIOUS v.9.0.5 (Kearse et

$157 a l ., 2012)$.

\section{Estimates of Divergence Times}

159 Our prior study suggested that including noncoding regions in phylogenetic analysis can

160 maximize the power to resolve relationships of Saussurea (Zhang, et al., 2019a). Whole

161 plastome sequences of 226 samples containing one inverted repeat region were aligned

162 using MAFFT v.7.22 (Katoh \& Standley, 2013). Poorly aligned regions were removed

163 with TRIMAL v.1.2 (Capella-Gutiérrez et al., 2009) using the command '-automated1'.

164 Age estimates were obtained using Markov Chain Monte Carlo (MCMC) analysis in

165 BEAST v.1.10.4 (Suchard et al., 2018). We used a GTR + I + $\Gamma$ nucleotide substitution

166 model, uncorrelated relaxed lognormal clock and a birth-death model for the tree prior

167 (Suchard et al., 2018). The MCMC analysis was run for 500 million generations,

168 sampling every 10,000 generations, resulting in 50,000 samples in the posterior

169 distribution of which the first 10,000 samples were discarded as burn-in. Convergence

170 and performance of the MCMC runs were checked using TRACER v.1.6 (Rambaut et al.,

171 2018). A maximum clade credibility (MCC) tree was then reconstructed in

172 TREEANNOTATOR v.1.8.4 (Rambaut \& Drummond, 2010), with median age and 95\%

173 height posterior density (HPD) annotated. Two high confident fossil calibrations with

174 lognormal distributions were assigned: (A) The crown age of Carduus-Cirsium group

175 was set to a minimum age of 14 million years ago (Mya) based on the Middle Miocene

176 achenes identified as Cirsium (Mai, 1995; Barres et al., 2013); (B) The split of Centaurea 
177 and Carthamus was calibrated with a minimum age of 5 Mya, based on the records of

178 pollen and achenes for Centaurea dating from the Early Pliocene (Popescu, 2002).

179 Additionally, the crown age of Cardueae was set to 39.2 Mya as a secondary calibration

180 with a normal distribution based on the estimation by Barres et al. (2013).

\section{Estimates of Diversification rate}

182 We explored the diversification dynamics of Saussurea using BAMM 2.5.0 (Rabosky,

183 2014), which employs a reversible-jump MCMC to sample a large number of possible

184 diversification regimes from a given time-calibrated phylogeny. We pruned the MCC tree

185 for BAMM analysis and retained only one sample of each species. Prior values were

186 selected using the 'setBAMMpriors' function in the R package BAMMtools v.2.1.7 (R

187 Core Team, 2014; Rabosky et al., 2014). Due to the controversial species number in

188 Saussurea, the incomplete taxon sampling was appropriately set as 0.5 for all following

189 analyses. The MCMC was run for 500 million generations and sampled every 50,000

190 generations. Post-run analyses were performed using the BAMMtools, with an initial

$19110 \%$ of the MCMC run discarded as burn-in, and the remaining data assessed for

192 convergence and ESS values $>200$. Rates-through-time plots were generated using

193 'PlotRateThroughTime' function for the entire genus as well as three clades. Speciation

194 rates of Saussurea species were obtained using the 'getTipRates' function. Considering

195 recent criticism relating to the statistical methods for lineage specific diversification

196 models like BAMM (Moore et al., 2016; but also see Rabosky et al., 2017), we also

197 employed the semiparametric DR statistic to calculate speciation rates, following the

198 method described in Jetz et al. (2012) and Sun et al. (2020). Analysis of variance

199 (ANOVA) was performed to determine whether differences among three phylogenetic

200 clades and among four traditional subgenera were significant. In addition, we used TESS

201 v.2.1 (Höhna et al., 2016) in R to detect the abrupt changes in speciation and extinction

202 rates, applying the R-scripts of Condamine et al. (2018). 


\section{Paleoenvironment dependent analyses}

204 To quantify the effects of past environmental conditions on Saussurea diversification, we 205 used RPANDA v1.9 (Condamine et al., 2013) to fit a series of time- and temperature206 dependent likelihood diversification birth-death (BD) models, following the methodology 207 of Condamine et al. (2018). Briefly, seven models were tested: BD model with constant $\lambda$ 208 (speciation rate) and $\mu$ (extinction rate) (i); BD model with $\lambda$ dependent to time (ii) and 209 environment (iii) exponentially, and constant $\mu$; BD model with constant $\lambda$, and $\mu$ 210 dependent to time (iv) and environment (v) exponentially; and BD model with $\lambda$ and $\mu$ 211 dependent to time (vi) and environment (vii) exponentially. Thus, we can obtain the 212 equations: $\lambda(E)=\lambda_{0} \times \mathrm{e}^{\alpha \mathrm{E}}$ and $\mu(\mathrm{E})=\mu_{0} \times \mathrm{e}^{\beta \mathrm{E}}$, in which $\lambda_{0}$ and $\mu_{0}$ are the speciation and

213 extinction rates for a given environmental variable. The values of $\alpha$ and $\beta$ are the rates of

214 change according to the environment, and positive values for them mean a positive effect

215 of the environment on speciation or extinction (Condamine et al., 2013). We used

216 paleotemperature over the last 12 million years (Myrs) (retrieved from Zachos et al.,

217 2008) as environmental variables, and randomly sampled 500 trees from the BEAST

218 posterior distribution (outgroups removed) to accommodate phylogenetic and dating

219 uncertainties. The R package PSPLINE v.1.0 (Ben \& Roberto, 2008) was used to

220 visualize the speciation rates variating with paleoenvironmental variables.

\section{Trait dependent analyses}

222 Nine characters were selected and coded based on descriptions in eFloras

223 (http://www.efloras.org/), herbarium specimens and taxonomic literature, or were

224 manually checked directly using online herbarium specimens from the Chinese Virtual

225 Herbarium (http://www.cvh.ac.cn/), JSTOR (https://plants.jstor.org/), and field collections

226 (Supporting Information Table S2). These characters included four binary morphological

227 traits: stemless (0) vs. cauliferous (1), stem glabrous (0) vs. densely haired (1), the 
228 absence (0) vs. presence (1) of leafy bracts, and capitula solitary (0) vs. numerous (1);

229 four multistate morphological traits: leaf margin entire (1) vs. pinnately lobed (2) vs. both

230 types (3), leaves glabrous (1) vs. sparsely haired (2) vs. densely haired (3), phyllary in $<5$

231 (1) vs. 5 (2) vs. 6 (3) vs. >6 (4) rows, and phyllary glabrous (1) vs. sparsely haired (2) vs.

232 densely haired (3) vs. appendage (4); as well as the geographical habitats: widespread (0)

233 vs. alpine (1) vs. lowland (2).

234 The diversification rate shifts of binary traits were investigated using the hidden state

235 speciation and extinction (HiSSE) model, which allows us to demonstrate hypotheses

236 related to both the effects of the observed traits as well as incorporate unmeasured factors

237 (Beaulieu \& O’Meara, 2016). As described in Beaulieu and O’Meara (2016), 25 models

238 were tested in the R package HISSE v.1.9.10: a full HiSSE model allowing all states to

239 vary independently; four binary state speciation and extinction (BiSSE)-like models that

240 excluded hidden states or constrained specific parameters of $\lambda, \mu$, and transition rates (q);

241 four null HiSSE models with various character-independent diversification (CID) forms;

242 and 16 models assuming a hidden state associated with both observed character states

243 with a variety of constrained values for $\lambda$, $\mu$, and q (Supporting Information Table S3).

244 The best-fitting model was selected based on likelihood-ratio tests under a Chi-square

245 distribution and Akaike's information criterion (AIC) (Akaike, 1974). We also used a

246 nonparametric FiSSE model (Fast, intuitive SSE model; Rabosky \& Goldberg, 2017)

247 serving as a complement to measure the robustness of our results. For multistate traits,

248 MuSSE analyses were performed in the R package DIVERSITREE v.0.9.10 (FitzJohn,

249 2012) by fitting four distinct models with subsequent ANOVA testing: a null model with

250 fully constrained variables; a full model allowing all variables to change independently; a

251 model constraining each $\mu$ to be equal (free $\lambda$ ); and a model constraining the $\lambda$ values for

252 each state to be equal (free $\mu$ ). Further estimates for the parameters of $\lambda$, $\mu$, and net

253 diversification rates $(\lambda-\mu)$ for each state were obtained in a Bayesian approach by 
254 incorporating a MCMC analysis with an exponential prior with 5,000 generations. A

255 GeoHiSSE analysis was used to test hypotheses about range-dependent diversification

256 processes (Caetano et al., 2018), implemented in the HISSE package. Two models with a

257 range-independent diversification process and two other models in which the range have

258 an effect on the diversification rate of the lineages were deployed and compared, as

259 described in Caetano et al. (2018).

\section{Ecological distribution data and association with diversification rates}

261 We used the 'occ_search' function of RGBIF v.1.3.0 (Chamberlain \& Boettiger, 2017) to

262 retrieve GPS coordinates for Saussurea species from GBIF on October 28, 2020. We

263 extracted only data records that were georeferenced and excluded any coordinates with

264 zero and/or integer latitude and longitude. We then removed geographic outliers defined

265 as boxplot outliers of species occurrences in R. Range size of each species was estimated

266 by applying a five kilometer buffer around each locality point using the 'gBuffer'

267 function of RGEOS v.0.5-5 (https://CRAN.R-project.org/package=rgeos) following the

268 descriptions of Testo et al. (2019). Range size data were log-transformed before analysis

269 to overcome their skewed distribution (Testo et al., 2019). We extracted the values of 19

270 bioclimatic variables (from 1970 to 2000) from WorldClim (http://worldclim.org) using

271 RASTER v.2.6-6 (https://CRAN.R-project.org/package=raster), and calculated a mean

272 value for each species. Highly correlated variables were identified with a Pearson's

273 correlation coefficient $>0.75$, and were removed. The remaining eight most predicative

274 bioclimatic variables were: annual temperature (BIO1), mean diurnal range (BIO2),

275 isothermality (BIO3), max temperature of warmest month (BIO5), annual precipitation

276 (BIO12), precipitation seasonality (BIO15), precipitation of warmest quarter (BIO18) and

277 precipitation of coldest quarter (BIO19). The main variation of bioclimatic variables

278 representing climate lability was estimated by extracting the first two principal

279 components (PC1 and PC2) from a PCA in R. To calculate the ecological niche breadth, 
280 we first estimated environmental niche models (ENM) in the R package ENMTOOLS

281 v.1.0.2 (Warren et al., 2010), and then measured the spatial heterogeneity of the

282 distribution of suitability scores using Levins' B metrics (Levins, 1968) ('raster.breadth'

283 function).

284 To demonstrate whether ecological factors drove rapid diversification of Saussurea

285 species, multiple QuaSSE tests were performed under different models using

286 DIVERSITREE. Five models with increasing complexity were constructed to fit the

287 changes in speciation rates with climate lability (PCs of bioclimatic variables), niche

288 breadth and species range size. Moreover, we used the ES-sim tests (Harvey \& Rabosky,

289 2018) to crosscheck the correlation pattern revealed by QuaSSE. In addition to the default

290 inverse equal splits statistic (Harvey \& Rabosky, 2018), the DR statistic was also used as

291 a reliable estimator to investigate correlation between speciation rate and continuous

292 ecological factors using the R-scripts retrieved from Sun et al. (2020).

\section{Data availability}

294 All newly sequenced plastomes were deposited in the National Center for Biotechnology

295 Information (NCBI) database with accession numbers provided in Supporting

296 Information Table S1. R scripts used in this study are available on GitHub

297 (https://github.com/ZhangXu-CAS/Saussurea-diversification.git). 


\section{Results}

\section{Divergence time and diversification rate}

300 Our phylogeny resolved a median stem age of ca. 11.79 Mya (95\% HPD, 8.38-15.35

301 Mya) for Saussurea, with three clades beginning to diversify in parallel during the

302 Miocene (ca. 9.05 Mya, ca. 8.37 Mya and ca. 8.92 Mya, respectively; Figs 1a, Supporting

303 Information Figs S1, S2), suggesting a rapid radiation in this period. Our tree topology

304 showed that four traditional morphology-based subgenera of Saussurea are paraphyletic,

305 indicating adaptive traits have occurred multiple times. BAMM analysis revealed a

306 scenario in which two shifts in net diversification rates occurred within Saussurea with

307 high posterior probability (Figs 1a, Supporting Information Figs S3). Rates-through-time

308 plots showed that while slightly offset in timing, diversification rates of the three clades

309 accelerated during the Pliocene (Figs 1a, 1b), when the temperature dropped sharply.

310 BAMM tip rates showed that clade-3 (0.981 events $\mathrm{Myr}^{-1}$ per lineage) had significantly

311 higher mean speciation rate than clade-2 ( 0.560 events $\mathrm{Myr}^{-1}$ per lineage) and clade-1

312 (0.708 events $\mathrm{Myr}^{-1}$ per lineage) $(p<0.001$, Supporting Information Tables S4, S5).

313 Among four morphological-based subgenera, speciation rates of S. subg. Amphilaena

314 (0.945 events $\mathrm{Myr}^{-1}$ per lineage) was highest $(p<0.001$, Fig 1c, Supporting Information

315 Tables S4, S5). While DR statistic revealed no significant difference among three main

316 clades $(p=0.099)$, and $S$. subg. Saussurea (1.106 events $\mathrm{Myr}^{-1}$ per lineage) have the

317 highest mean speciation rate ( $p=0.022$, Supporting Information Fig S4, Tables S4, S6).

318 TESS analysis suggested that speciation and exaction shifts had higher posterior

319 probability during the Pleistocene, consistent with the BAMM results (Supporting

320 Information Fig S5). 
(a)

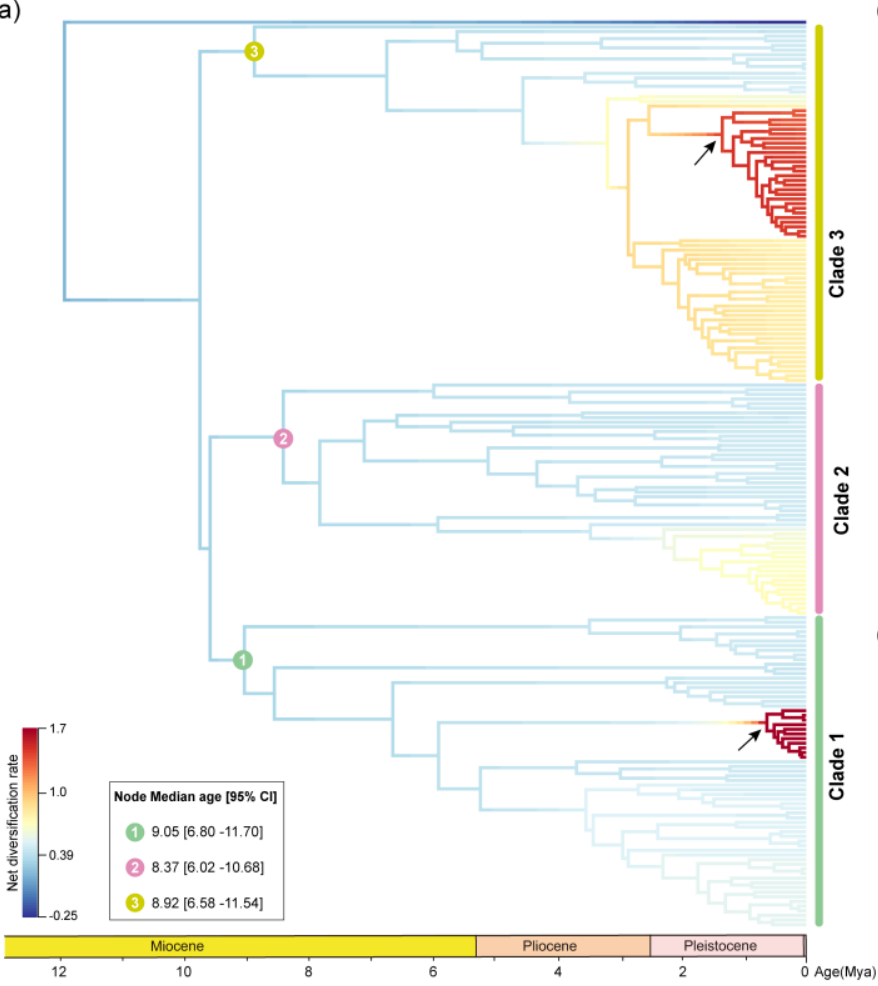

(b)

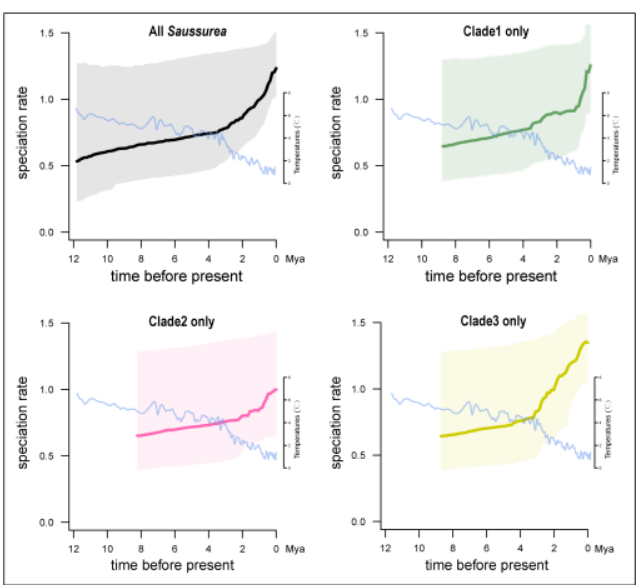

(c)

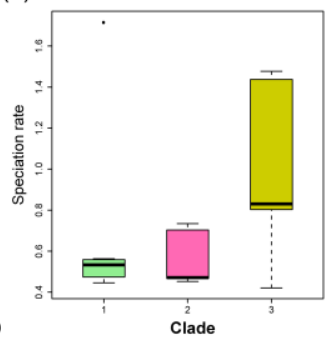

(d)

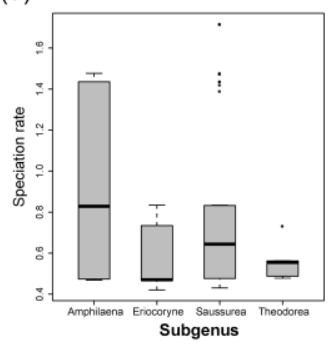

322 Fig. 1 Diversification dynamics of Saussurea inferred from BAMM analysis. (a) BAMM identified

323 two shifts in diversification rates (represented by arrows). The time of three clades beginning to

324 diversify is provided. (b) Rates-through-time plots of all Saussurea species and three main clades

325 separately, with trends in global climate change over 12 million years (Zachos et al. 2008) depicted.

326 (c-d) BAMM tip rates of three clades and four morphology-based subgenera of Saussurea,

327 respectively.

328 Paleoenvironment dependent diversification

329 We used a maximum-likelihood framework to illustrate diversification dynamics

330 dependent to paleoenvironment based on BD models to gain insight into the role of

331 historical processes on diversification. Out of seven models, a model with temperature-

332 dependent speciation fit the data best (Table 1). The best-fit model further indicated a

333 negative dependence $(\alpha<0)$ between past temperature and speciation rate for Saussurea,

334 while extinction rate remained constant, suggesting extinction was likely not affected by

335 temperature fluctuations. RPANDA results demonstrated a diversification regime in

336 which diversification rates had opposite responses to changes of temperature over time, 
337 and accelerated sharply in the Pleistocene and increased toward the present (Fig. 2),

338 consistent with the conclusion of rates-through-time in BAMM analysis (Fig. 1b).

(a) Global paleo-temperatures over the last 12 Myrs

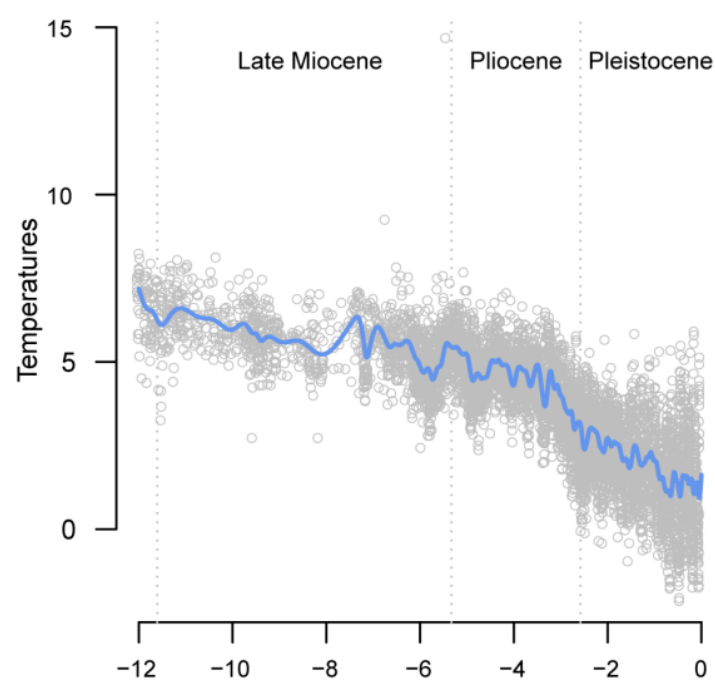

(b) Speciation is negatively correlated to past temperatures

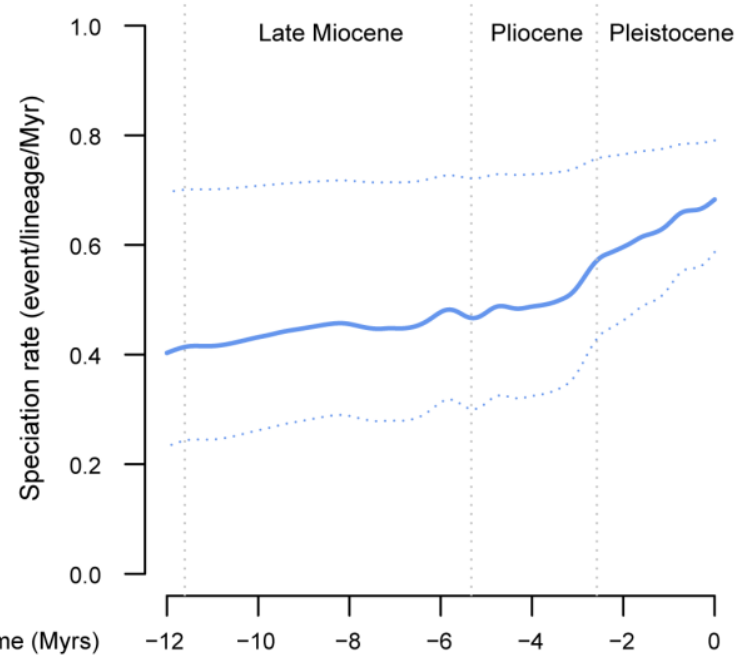

339 Fig. 2 Paleoenvironment-dependent diversification processes in Saussurea. The best-fit

340 paleoenvironment-dependent model implemented in RPANDA shows negative dependence

341 between paleotemperatures (a) and speciation rate (b).

\section{Trait dependent diversification}

343 We investigated eight morphological characters and geographical habitat that serve as a

344 proxy for the effect of adaptive traits on diversification rate, to understand the role of trait

345 innovations in the rapid radiation of Saussurea. For all four binary traits, the best model

346 of the 25 models tested was the full HiSSE model with unique speciation, extinction and

347 transition rates between the two character states observed and the hidden states

348 (Supporting Information Tables S7). We then calculated mean speciation, extinction and

349 net diversification rates values from the model-averaged marginal ancestral state

350 reconstruction for each extant species in our tree. The results suggested that species with

351 cauliferous plant, glabrous stem, leafy bracts and solitary capitula have higher mean

352 speciation, extinction and net diversification (Table 2, Fig. 3). While the full HiSSE

353 model showed observed differences in diversification rates between the states of these 
354 traits, it also indicated some unobserved traits drive the diversification. The

355 complementary results from our FiSSE analysis supported the tendency of speciation rate

356 revealed by HiSSE, but the only significant differences were between solitary capitula

357 and numerous capitula (Table $2 ; p=0.024$ ).
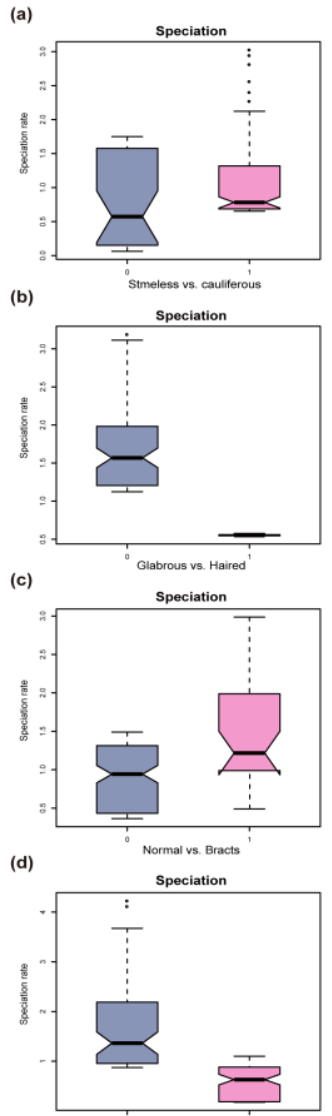

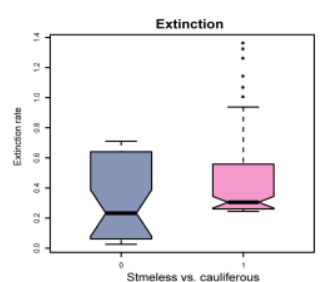

Extinction

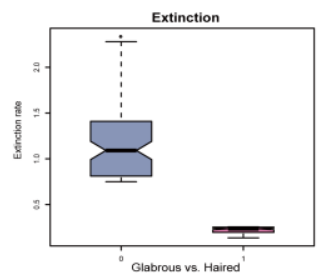

Extinction

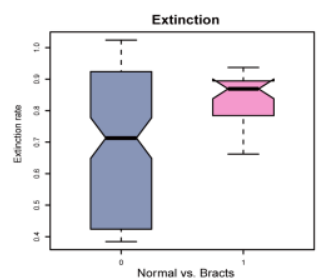

Extinction

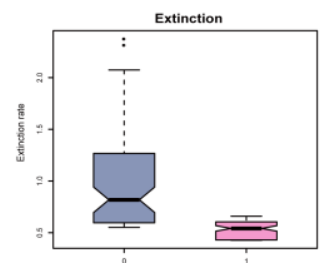

'Soltany vs Numerout
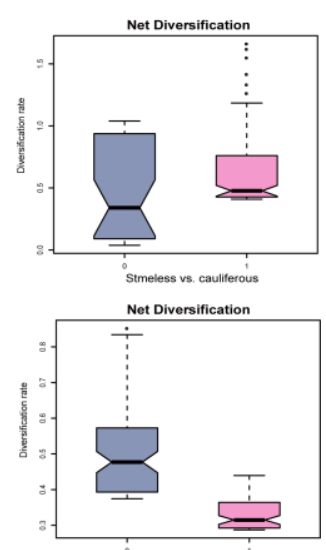

Fig. 3 Binary trait dependent diversification of Saussurea inferred from HiSSE analysis. Speciation, extinction and net diversification rates are calculated by the model-averaged marginal ancestral state reconstruction for four binary traits: (a) stemless (0) vs. cauliferous (1), (b) stem glabrous (0) vs. densely haired (1), (c) the absence (0) vs. presence (1) of leafy bracts, and (d) capitula solitary (0) vs. numerous (1).

374 In the MuSSE analyses, ANOVA calculations all preferred models constraining each

$375 \mu$ to be equal and allowing $\lambda$ to vary (free $\lambda$ ), compared with either null models and full

376 models (Supporting Information Table S8). The best-fitting model was then used as the

377 starting point for a MCMC run of 5,000 generations to estimate the marginal distributions

378 of rates for each traits using a state-dependent model (Fig. 4). Since all the models

379 preferred constrained $\mu$ values, all of the estimated probability densities of $\mu$ overlapped

380 (data not shown). The reconstructions of probability density of the net diversification

381 rates $(\lambda-\mu)$ showed that some traits, i.e. leaf margin and phyllary types, have an overlap 
382 in net diversification rates among examined character states (Fig. 4a, d). The results

383 suggest that species with glabrous leaves have higher net diversification rates than

384 sparsely or densely haired species, consistent with higher mean rates for glabrous stem in

385 the HiSSE analysis. For the phyllary character, the glabrous state also showed higher net

386 diversification rates than sparsely or densely haired states despite some overlapping (Fig.

$3874 d$ ), and the rates of phyllary with six rows are higher than the remaining character states

388 (Fig. 4c). The GeoHiSSE analysis suggested a model with one hidden area and no range-

389 dependent diversification was the best fitting model (Supporting Information Table S9).

390 From the result, we can see that species with lowland habitats have a substantially higher

391 speciation, extinction and net diversification rates in comparison with both alpine and

392 widespread distributions (Supporting Information Fig. S6).

(a)

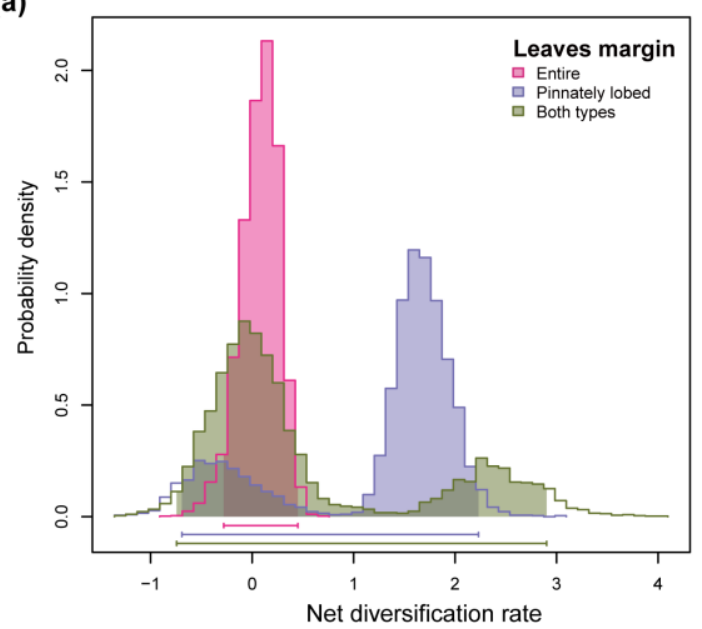

(c)

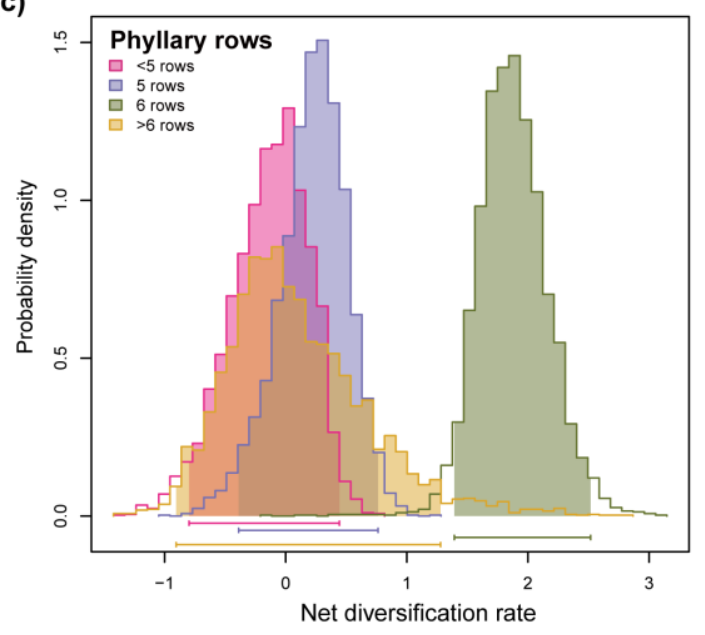

(b)

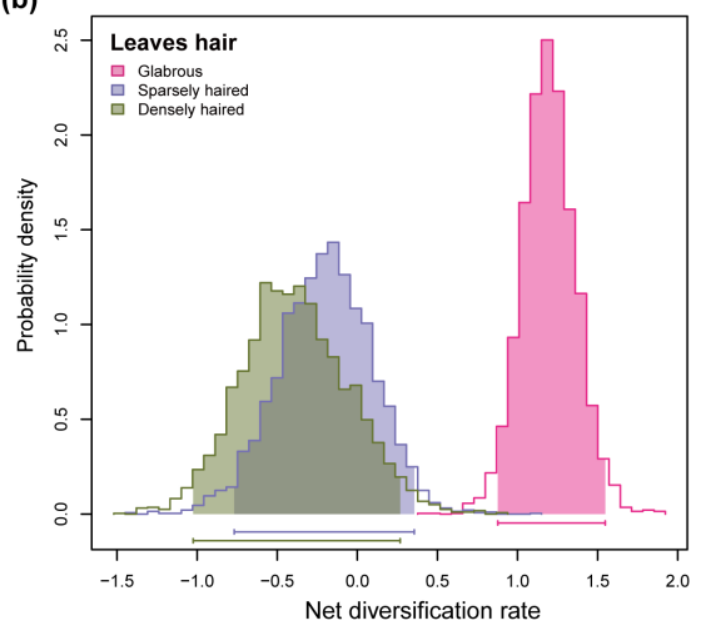

(d)

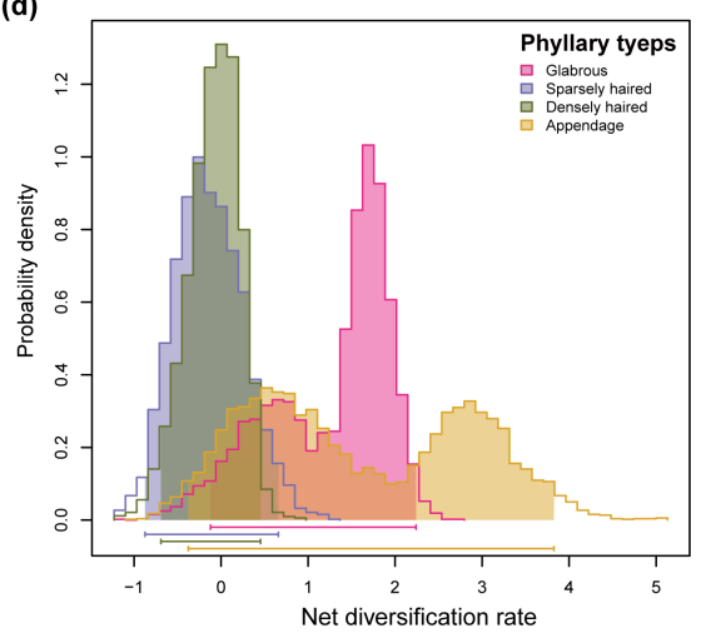


393 Fig. 4 Multistate trait dependent diversification of Saussurea estimated from MuSSE analysis.

394 Marginal distributions of net diversification rates are estimated by the MCMC run of 5, 000

395 generations for four multistate traits: (a) leaves margin entire (1) vs. pinnately lobed (2) vs. both types

396 (3), (b) leaves glabrous (1) vs. sparsely haired (2) vs. densely haired (3), (c) phyllary in <5 (1) vs. 5 (2)

397 vs. 6 (3) vs. >6 (4) rows, and (d) phyllary glabrous (1) vs. sparsely haired (2) vs. densely haired (3) vs.

398 appendage (4).

(a)

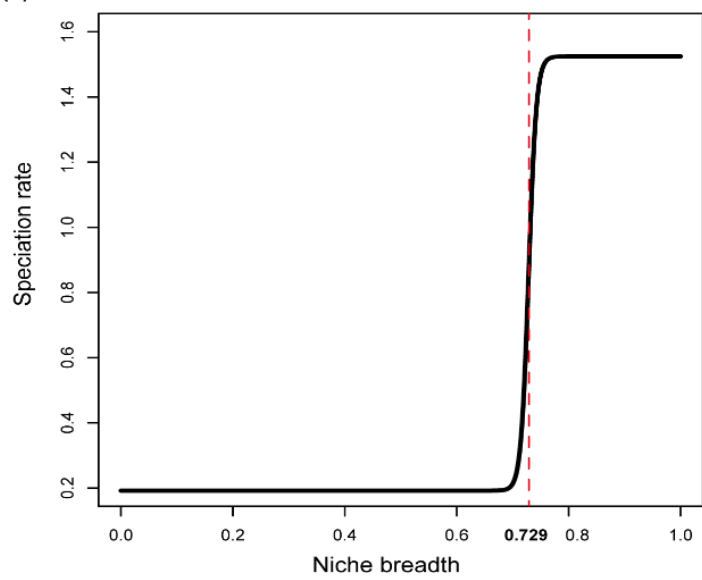

(c)

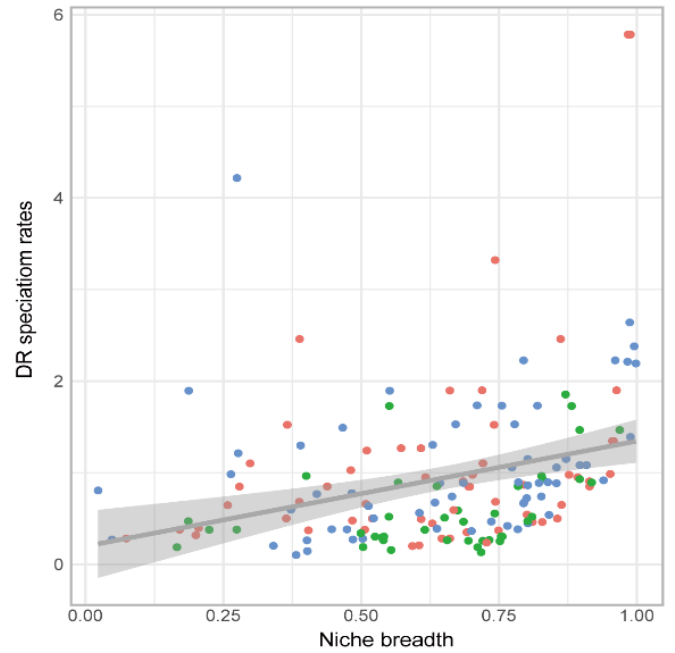

(b)

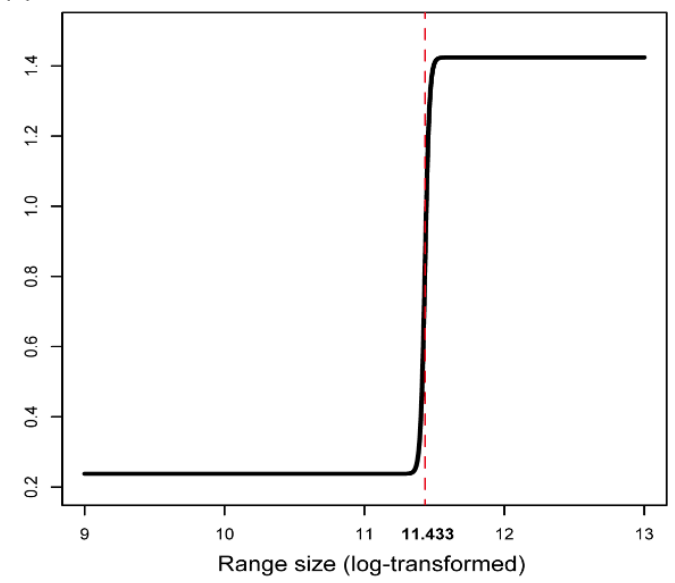

(d)

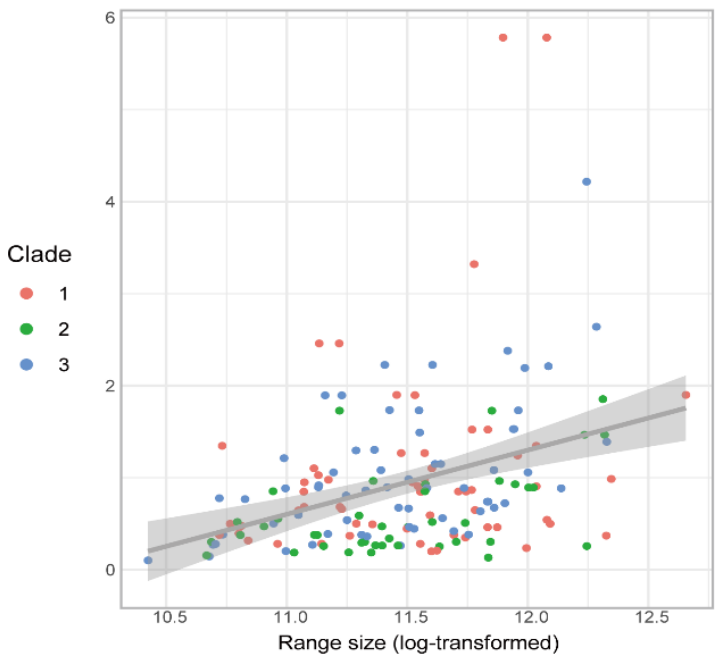

399 Fig. 5 Speciation rates of Saussurea correlated with ecological factors based on the QuaSSE best-

400 fitted model and ES-sim tests. Both (a) niche breadth and (b) species range size (log-transformed)

401 show positive sigmoidal curves in QuaSSE analysis with the midpoints (represented by the red dashed

402 line) of 0.729 and 11.433 on the x-axis respectively. EM-sim tests show significant positive

403 relationships between DR speciation rates and (c) niche breadth and (d) species range size. Species

404 from three clades are in different colors. 


\section{Ecological drivers of diversification}

406 By correlating climate lability (PCs of bioclimatic variables), niche breadth and species

407 range size with speciation rates (Supporting Information Table S10), we explored the role

408 of ecological opportunities created by complex QTP environments in driving

409 diversification of Saussurea. The first two PCs of bioclimatic variables explained 75.7\%

410 of the total climate variation in Saussurea (Supporting Information Fig. S7a). Among the

411 eight retained bioclimatic variables, the precipitation of warmest quarter (BIO18) had the

412 largest contribution to first two PCs, followed by the annual precipitation (BIO12) and

413 the mean diurnal range (BIO2) (Supporting Information Fig. S7b). Under the QuaSSE

414 analyses, PC1 of the climate variables showed a significant positive linear $(1 . \mathrm{m}=0.330$,

$415 \mathrm{AIC}=1240.548, p$-value $\left.=0.005^{* *}\right)$ relationships with speciation rate, while climate

416 PC2 preferred a constant model $($ AIC $=1183.524, p$-value $=0.953)$; both niche breadth

$417\left(\mathrm{AIC}=529.532, p\right.$-value $\left.<0.000^{* *}\right)$ and species range size $(\mathrm{AIC}=700.671, p$-value $<$

$4180.000^{* *}$ ) showed a significant positive sigmoidal (with drift) relationships with

419 speciation rate (Supporting Information Table S11). Under the best sigmoidal models, the

420 speciation rates of Saussurea kept a stable low state until the niche breadth and

421 distribution range reached at 0.729 and 11.433 (log-transformed), respectively

422 (midpoints; Fig. 5a, 5b). Under the EM-sim tests, both the DR statistic and the default

423 inverse equal splits statistic revealed the same correlation pattern, in which niche breadth

$424(\rho=0.363$ and $0.387, p=0.027$ and 0.019$)$ and range size $(\rho=0.399$ and $0.411, p=$

4250.018 and 0.011) showed significant positive relationship with speciation rates (Fig. 5c,

$4265 \mathrm{~d}$ ), while the correlation between speciation rates and climate lability (climate PC1: $\rho=$

4270.170 and $0.188, p=0.359$ and 0.335 ; climate PC2: $\rho=0.098$ and $0.095, p=0.649$ and

428 0.635) was not significant (Table 3). 


\section{Discussion}

430 Our results demonstrate rapid diversification of Saussurea occurred in parallel during the

431 Miocene, a period with extensive tectonic movement and climatic fluctuation on the QTP.

432 A recent paper by (Louca \& Pennell, 2020) raised limitations of macroevolutionary

433 studies using estimated diversification rates, though several recent papers have suggested

434 that more complex models (such as hidden state SSE models; (Helmstetter et al., 2021)

435 and a hypothesis driven approach (Morlon et al., 2020) circumvent many of the issues

436 raised. Therefore, we took an integrative approach to address the role that morphological

437 traits and environmental conditions played in the evolutionary history of Saussurea. The

438 rates of species diversification are revealed to be negatively correlated with

439 paleotemperature, and accelerate sharply in the Pliocene toward the present. Similar

440 patterns of increased diversification with global cooling have been documented in other

441 flowering plant lineages, e.g. Saxifragales (Folk et al., 2019), rosids (Sun et al., 2020)

442 and Campanulaceae (Lagomarsino et al., 2016), as well as in mammals (Stadler, 2011)

443 and birds (Claramunt \& Cracraft, 2015). Our trait dependent models detect some

444 observed phenotypic adaptation associated with diversification changes, and indicate

445 some unobserved traits also drive diversification, demonstrating a pivotal role of

446 morphological diversity in this radiating diversification. Accounting for ecological niche

447 data, we further reveal that acceleration in diversification rates are correlated with climate

448 lability (PCs of bioclimatic variables), niche breadth and the size of species' range.

449 Overall, we conclude that tectonic activity of the QTP along with global paleoclimate

450 cooling provided vast alpine niches for Saussurea species with ample adaptive traits,

451 highlighting the important role of morphological diversity and ecological niche

452 availability for species radiating to diverse environments.

453 We determined clade ages across Saussurea species using whole plastome sequences

454 and found that the divergence of the main species clades occurred in the Miocene almost 
455 simultaneously. Compared to fragment DNA markers, plastomes have been shown to

456 provide more sufficient phylogenetic signals which are powerful in resolving deep

457 relationships of plant lineages (Parks et al., 2009; Wicke et al., 2011; Zhang et al., 2020).

458 Our estimate for the origin of Saussurea (ca. 11.8 Ma) is consistent with the result from

459 single-copy nuclear genes obtained via Hyb-Seq (ca. 12.5 Mya) (Herrando-Moraira et al.,

460 2019) and the result from ITS sequences (12.6-10.3 Mya) (Wang et al., 2009), but was

461 younger than the result of Xu et al. (2019) (ca. 18.5 Mya) using plastome coding regions

462 and the result of Barres et al. (2013) (ca. 20.0 Mya) using chloroplast markers. The study

463 of Barres et al. (2013) included only two species of Saussurea and used four chloroplast

464 markers, trnL-trnF, matK, $n d h F$ and $r b c L$. Different from Xu et al. (2019) setting the split

465 of subtribe Arctiinae and subtribe Saussureinae as a minimum age to 8.0 Mya using the

466 achene fossil assigned to Arctium, our study omitted this calibration because only one

467 Arctiinae sample (A. lappa) was included in both studies and the relationship between

468 Arctiinae and Saussureinae remains unresolved (Herrando-Moraira et al., 2019; Shen et

$469 a l ., 2020)$. In addition, we estimated divergence times using whole plastome sequences,

470 as our prior work showed that including noncoding regions can maximize the resolution

471 in resolving relationships of Saussurea (Zhang et al., 2019a).

472 Recent large-scale studies of species diversification on the QTP have provided

473 convincing evidence for a Miocene diversification in plant lineages (Ding et al., 2020) as

474 well as amphibians and reptiles (Xu et al., 2020). A hypothesis for the rich biodiversity

475 found in mountainous regions like the QTP is uplift-driven diversification - that orogenic

476 activities create diverse habitats favoring rapid in situ speciation of resident lineages

477 (Xing \& Ree, 2017; Chen et al., 2019). Extensive plateau uplift in the Miocene further

478 intensified the Asian summer monsoon, which increased the precipitation for erosion

479 through river incision, leading to greater topographic relief (Nie et al., 2018). This would

480 have promoted the differentiation of microhabitats associated with elevational gradients 
481 and slope aspects, increasing the availability of ecological niches for radiating species

482 (Ding et al., 2020). A previous study indicated that the Saussurea radiation was likely

483 driven by ecological opportunities, similar to those on islands, provided by largely

484 unoccupied habitats resulting from the extensive QTP uplifts (Wang et al., 2009). Our

485 work provides compelling evidence of the vital role of ecological opportunities in

486 Saussurea diversification by statistically correlating species niche breadth and

487 distribution range to the speciation rate. A slight difference is that our result supports a

488 wide-range radiation rather than an 'island isolation', from the positive correlation

489 between range and speciation rate. We attribute the wide-range radiation of Saussurea to

490 the presence of unique pappus combinations (Shi \& Raab-Straube, 2011; Chen, 2015),

491 which can promote the dispersal power of achenes to occupy more newly created niches.

492 Therefore, colonizing success benefited by wide-range dispersal helped Saussurea

493 species become one of the most diverse lineages on the QTP.

The negative correlation between paleotemperature and diversification rates in

495 Saussurea does not seem surprising given the high species richness of Saussurea found at

496 the high elevations of the QTP. Nonetheless, this insight is progressive for our

497 understanding of the formation of the QTP flora, as it represents one of the few attempts

498 to explicitly quantify the relationship between lineage diversification and a

499 paleoenvironmental variable. Geological evidence suggests that after 15 Mya, global

500 cooling and the further rise of QTP progressively led to more open, herb-rich vegetation

501 as the modern high plateau formed with its cool, dry climate (Spicer et al., 2021). Thus,

502 diversification among Saussurea clades could have been driven by increased ecological

503 niches as suitable cold habitats became available. A sharply accelerated diversification

504 rate of Saussurea was detected in the Pliocene toward the present. The uplift of the

505 Hengduan Mountains region, at the southeastern margin of the QTP, is generally believed

506 to have been rapid and recent, occurring mainly between the late Miocene and late 
507 Pliocene (Xing \& Ree, 2017; Spicer et al., 2020). During the Quaternary glaciation, the

508 Hengduan Mountains with its deep valleys would have provided numerous micro-refugia

509 within the altitudinal and aspect heterogeneity (Sun et al., 2017; Spicer et al., 2021). This

510 can explain why extensive morphological traits occur in parallel and evolved

511 convergently, a result likely driven by local adaptation to the micro-habitats that were

512 afforded by the complex and highly dissected landscape of the Hengduan Mountains.

513 Trait dependent analyses demonstrated that species exhibiting cauliferous plant,

514 glabrous stem, leafy bracts and solitary capitula have higher speciation rates. These traits

515 are usually observed in the subgenus Amphilaena (snow lotus), which is characterized by

516 attractive leafy bract and is the symbols of snow mountains in the QTP (Shi \& Raab-

517 Straube, 2011; Chen, 2015). Snow lotus has abundant morphological variation and is a

518 taxonomically complex group, with some new species described recently (e.g. Eckhard

519 von, 2009; Chen \& Yuan, 2015; Zhang, et al., 2019b). Despite having significant

520 taxonomic characteristics, snow lotus is a non-monophyletic group, demonstrating that

521 these adaptive traits have multiple origins and arose by convergent evolution. In fact,

522 specialized leafy bracts, the so-called 'glasshouse' morphology, are prevalent among

523 alpine species, such as in Lamiaceae, Asteraceae, and Polygonaceae (reviewed by Sun et

$524 a l ., 2014)$. Leafy bracts reportedly protect pollen grains from damage by UV-B radiation

525 and rain, promote pollen germination by maintaining warmth, enhance pollinator

526 visitation by providing a vivid visual display during flowering, and facilitate the

527 development of fertilized ovules during seed development (Tsukaya, 2002; Yang \& Sun,

528 2009; Song et al., 2015). Convergent morphological evolution seems to be common for

529 plants adapting harsh environments of the QTP, examples include cushion (stemless)

530 plant, woolly hairs and the leafy bract (Sun et al., 2014; Peng et al., 2015; Yang et al.,

531 2019). Similar to leafy bract, the present of stemless and woolly hairs has been revealed

532 to occurred multiple times, and is thought to defense cold and arid climate on the plateau 
533 (Sun et al., 2014). However, both stemless plants and the presence of woolly hairs appear

534 to be not associated with an increase in diversification rate of Saussurea. A plausible

535 explanation for this is that species with stemless and woolly hairs are commonly found in

536 environments of the QTP with extremely high altitude with very low temperature, and

537 these species usually have long lifespans.

538 Some traits associated with high diversification rates appear to have no evidence for

539 ecological adaptation, such as solitary capitula and pinnate leaf margin. These may occur

540 in combination with other important adaptive traits. Some traits were not examined

541 because they are common across the entire genus, such as two rows of pappus and small

542 achenes (Shi \& Raab-Straube, 2011; Chen, 2015). Although trait dependent analyses

543 showed several adaptive traits driving the increase of speciation rate, some unobserved

544 traits were also important for rapid diversification, highlighting the vital roles of

545 morphological diversity in the evolutionary history of Saussurea. Morphological

546 diversity is an essential but often neglected aspect of biodiversity (Chartier et al., 2021).

547 Our work provides a valuable guide for conservation efforts in the protection of

548 morphological diversity of organisms, especially in the context of exacerbated

549 biodiversity loss due to global warming.

550 Our results provided evidence of a positive relationship between speciation rate and 551 niche breadth as well as species range. Among the few studies that have tested a niche 552 breadth-diversification relationship, a clear consensus has not been reached (Sexton et 553 al., 2017). One argument for low niche breadth lineages having greater diversification

554 rates is that they are more likely to suffer from resource limitations and more susceptible 555 to range fragmentation, and thus allopatric speciation occurs more frequently (Vrba, 556 1987). An alternative view is that species with high niche breadth typically have larger 557 range sizes (Slatyer et al., 2013) and are therefore more likely to have these ranges 558 fragmented by ecological or geographical barriers over evolutionary time, promoting 
559 allopatric speciation (Rolland \& Salamin, 2016). We argue that wider ecological niches

560 can help species diverging in the QTP cope with climatic fluctuation, occupy

561 microhabitats and promote morphological divergence. Note that anthropogenic activities

562 have led to landscape modification and habitat fragmentation, alternating the distributions

563 of a vast array of species (Boivin et al., 2016), even in plateau areas (Chen et al., 2014).

564 To promote future biodiversity resilience, the conservation of entire unfragmented

565 landscapes is necessary to preserve niche heterogeneity and enable species migrations at

566 will. Only this approach will conserve the processes of biodiversity dynamics as well as

567 the genetic library and the capacity for future adaptation in threatened species (Spicer et

568 al., 2020).

\section{Conclusion}

570 Despite substantial processes on the taxonomy, phylogeny and biogeography of plant

571 lineages on the QTP (reviewed by Wen et al., 2014), our knowledge of the diversification

572 rates associated with geological activities along with subsequent environmental

573 fluctuations and biotic interactions is still limited, especially for rapidly radiating species.

574 Our study integrates Saussurea into an marcoevolutionary diversification framework.

575 Using a genomic data set (plastome sequences) for reconstructing divergence history and

576 multiple statistical analyses, we quantify the roles of abiotic/environmental and

577 biotic/species-intrinsic factors in driving diversification of Saussurea. Our comprehensive

578 and large-scale analyses depict a plausible evolutionary scene for Saussurea, and provide

579 insights into the drivers of its radiating diversification. We document a Miocene

580 diversification pattern in which increased speciation rates are related to global cooling,

581 and correlate it to clade-specific traits and ecological niches. We hypothesize that the

582 current mega diversity of Saussurea is the result of interactions between geological

583 activity, global paleoclimate and ecological niche. Our results highlight the vital roles of

584 morphological diversity and available ecological niches in plants adapting to the 
585 changing climate. Given the ongoing global warming and human expansion, causing the

586 disappearance of numerous undescribed species and extensive occupied habitats, our

587 present study together with previous macroevolutionary pattern studies (e.g. Condamine

588 et al., 2018; Folk et al., 2019; Testo et al., 2019; Ding et al., 2020; Sun et al., 2020)

589 provide valuable theoretical basis for mitigating the threats posed to biodiversity.

\section{Acknowledgements}

591 We are grateful to all the collectors of Saussurea morphological data. We thank Ting-

592 Shen Han for helpful in the visualization of Fig.5. We thank Jun-tong Chen and Kai Xue

593 for providing the photos of Saussurea species. We also thank the members of the alpine

594 research group of KIB, Jianwen Zhang, Zhuo Zhou, Hongliang Chen, Lishen Qian, Lu

595 Sun and Yongzeng Zhang for helping with sample collection, and Yazhou Zhang for

596 helping with species identification. This study was supported by the Second Tibetan

597 Plateau Scientific Expedition and Research (STEP) program (2019QZKK0502), the

598 Strategic Priority Research Program of Chinese Academy of Sciences (XDA20050203),

599 the Key Projects of the Joint Fund of the National Natural Science Foundation of China

600 (U1802232), the Major Program of the National Natural Science Foundation of China

601 (31590823), the Youth Innovation Promotion Association of Chinese Academy of

602 Sciences (2019382), the Young Academic and Technical Leader Raising Foundation of

603 Yunnan Province (2019HB039), the Chinese Academy of Sciences "Light of West China"

604 Program, and the Biodiversity Survey, Monitoring and Assessment

605 (2019HB2096001006), and the International Partnership Program of Chinese Academy of

606 Sciences (151853KYSB20180009).

\section{Author contributions}

608 HW, HS, TD and XZ developed the idea and designed the experiment; XZ and JBL

609 performed the statistical analyses; XZ, JBL, TD, HS and HW interpreted the results and 
610 wrote the manuscript. XZ, YS, TF, HZ, NL, TB, XH and TD collected the leaf materials;

611 All authors read, edited and approved the final manuscript.

\section{References}

613 Akaike H. 1974. A new look at the statistical model identification. IEEE Transactions on

$614 \quad$ Automatic Control 19: 716-723.

615 Antonelli A. 2015. Multiple origins of mountain life. Nature 524: 300-301.

616 Antonelli A, Kissling WD, Flantua SGA, Bermúdez MA, Mulch A, Muellner-Riehl AN,

617 Kreft H, Linder HP, Badgley C, Fjeldså J, et al. 2018. Geological and climatic influences

618 on mountain biodiversity. Nature Geoscience 11: 718-725.

619 Barres L, Sanmartin I, Anderson CL, Susanna A, Buerki S, Galbany-Casals M, Vilatersana

620 R. 2013. Reconstructing the evolution and biogeographic history of tribe Cardueae

621 (Compositae). American Journal of Botany 100: 867-882.

622 Beaulieu JM, O'Meara BC. 2016. Detecting hidden diversification shifts in models of traitdependent speciation and extinction. Systematic Biology 65: 583-601.

Ben J, Roberto GG 2008. "PSPLINE: Stata module providing a penalized spline scatterplot smoother based on linear mixed model technology," Statistical Software Components S456972, Boston College Department of Economics, revised 25 Jan 2009. term anthropogenic shaping of global species distributions. Proceedings of the National Academy of Sciences 113: 6388.

Caetano DS, O'Meara BC, Beaulieu JM. 2018. Hidden state models improve state-dependent diversification approaches, including biogeographical models. Evolution 72: 2308-2324.

Capella-Gutiérrez S, Silla-Martínez JM, Gabaldón T. 2009. trimA1: a tool for automated alignment trimming in large-scale phylogenetic analyses. Bioinformatics 25: 1972-1973.

Chamberlain SA, Boettiger C. 2017. R Python, and Ruby clients for GBIF species occurrence data. PeerJ Preprints 5: e3304v3301.

Chartier M, von Balthazar M, Sontag S, Löfstrand S, Palme T, Jabbour F, Sauquet H, Schönenberger J. 2021. Global patterns and a latitudinal gradient of flower disparity: perspectives from the angiosperm order Ericales. New Phytologist https://doi.org/10.1111/nph.17195.

Chen B, Zhang X, Tao J, Wu J, Wang J, Shi P, Zhang Y, Yu C. 2014. The impact of climate change and anthropogenic activities on alpine grassland over the Qinghai-Tibet Plateau. Agricultural and Forest Meteorology 189-190: 11-18. 2019. Genome-wide analysis of Cushion willow provides insights into alpine plant divergence in a biodiversity hotspot. Nature Communications 10: 5230. 
647 Chen Y-S, Deng T, Zhou Z, Sun H. 2018. Is the East Asian flora ancient or not? National

$648 \quad$ Science Review 5: 920-932.

649 Chen YS 2015. Asteraceae II Saussurea. In: Hong D-Y, Sun H, Watson M, Wen J, Zhang X-C

650 eds. Flora of Pan-Himalaya. Beijing: Science Press.

651 Chen YS, Yuan Q. 2015. Twenty-six new species of Saussurea (Asteraceae, Cardueae) from the

652 Qinghai-Tibetan Plateau and adjacent regions. Phytotaxa 213: 159-211.

653 Claramunt S, Cracraft J. 2015. A new time tree reveals Earth history's imprint on the evolution

654 of modern birds. Science Advances 1: e1501005.

655 Condamine FL, Rolland J, Höhna S, Sperling FAH, Sanmartín I. 2018. Testing the role of the

656 red queen and court jester as drivers of the macroevolution of apollo butterflies. Systematic

657 Biology 67: 940-964.

658 Condamine FL, Rolland J, Morlon H. 2013. Macroevolutionary perspectives to environmental

659 change. Ecology Letters 16: 72-85.

660 Dierckxsens N, Mardulyn P, Smits G. 2017. NOVOPlasty: de novo assembly of organelle

661 genomes from whole genome data. Nucleic Acids Res 45: e18.

662 Ding W-N, Ree RH, Spicer RA, Xing Y-W. 2020. Ancient orogenic and monsoon-driven

663 assembly of the world's richest temperate alpine flora. Science 369: 578.

664 Drummond CS, Eastwood RJ, Miotto STS, Hughes CE. 2012. Multiple Continental

665 Radiations and Correlates of Diversification in Lupinus (Leguminosae): Testing for Key

666 Innovation with Incomplete Taxon Sampling. Systematic Biology 61: 443-460.

667 Eckhard von R-S. 2009. Saussurea luae (Compositae, Cardueae), a new species of Snow Lotus

668 from China. Willdenowia 39: 101-106.

669 Favre A, Päckert M, Pauls SU, Jähnig SC, Uhl D, Michalak I, Muellner-Riehl AN. 2015. The

670 role of the uplift of the Qinghai-Tibetan Plateau for the evolution of Tibetan biotas. Biological

671 Reviews 90: 236-253.

672 FitzJohn RG. 2012. DIVERSITREE: comparative phylogenetic analyses of diversification in R.

673 Methods in Ecology and Evolution 3: 1084-1092.

674 Folk RA, Stubbs RL, Mort ME, Cellinese N, Allen JM, Soltis PS, Soltis DE, Guralnick RP.

675 2019. Rates of niche and phenotype evolution lag behind diversification in a temperate

676 radiation. Proceedings of the National Academy of Sciences 116: 10874. shifts. New Phytologist 225: 571-583. Xing Y-W. 2020. Polyploidy promotes species diversification of Allium through ecological

Harvey MG, Rabosky DL. 2018. Continuous traits and speciation rates: Alternatives to statedependent diversification models. Methods in Ecology and Evolution 9: 984-993.

684 Lineages-Through-Time Plots and Modern Macroevolutionary Modelling. bioRxiv:

$685 \quad 2021.2001 .2004 .424672$. 
Herrando-Moraira S, Calleja JA, Galbany-Casals M, Garcia-Jacas N, Liu JQ, Lópezand plastid DNA phylogeny of tribe Cardueae (Compositae) with Hyb-Seq data: A new subtribal classification and a temporal diversification framework. Molecular Phylogenetics and Evolution 137: 313-332.

Höhna S, May MR, Moore BR. 2016. TESS: an R package for efficiently simulating phylogenetic trees and performing Bayesian inference of lineage diversification rates. Bioinformatics 32: 789-791.

Howard CC, Landis JB, Beaulieu JM, Cellinese N. 2020. Geophytism in monocots leads to higher rates of diversification. New Phytologist 225: 1023-1032.

Hughes CE, Atchison GW. 2015. The ubiquity of alpine plant radiations: from the Andes to the Hengduan Mountains. New Phytologist 207: 275-282.

Jetz W, Thomas GH, Joy JB, Hartmann K, Mooers AO. 2012. The global diversity of birds in space and time. Nature 491: 444-448.

Katoh K, Standley DM. 2013. MAFFT multiple sequence alignment software version 7: improvements in performance and usability. Molecular Biology and Evolution 30: 772-780.

Kearse M, Moir R, Wilson A, Stones-Havas S, Cheung M, Sturrock S, Buxton S, Cooper A, Markowitz S, Duran C, et al. 2012. Geneious Basic: an integrated and extendable desktop software platform for the organization and analysis of sequence data. Bioinformatics 28: 16471649.

Kita Y, Fujikawa K, Ito M, Ohba H, Kato M. 2004. Molecular phylogenetic analyses and systematics of the genus Saussurea and related genera (Asteraceae, Cardueae). Taxon 53: 679690.

\section{Koenen EJM, Ojeda DI, Steeves R, Migliore J, Bakker FT, Wieringa JJ, Kidner C, Hardy} OJ, Pennington RT, Bruneau A, et al. 2020. Large-scale genomic sequence data resolve the deepest divergences in the legume phylogeny and support a near-simultaneous evolutionary origin of all six subfamilies. New Phytologist 225: 1355-1369.

Lagomarsino LP, Condamine FL, Antonelli A, Mulch A, Davis CC. 2016. The abiotic and biotic drivers of rapid diversification in Andean bellflowers (Campanulaceae). New Phytologist 210: 1430-1442.

724 Linder HP, Verboom GA. 2015. The Evolution of Regional Species Richness: The History of 
the Southern African Flora. Annual Review of Ecology, Evolution, and Systematics 46: 393-

412.

Louca S, Pennell MW. 2020. Extant timetrees are consistent with a myriad of diversification histories. Nature 580: 502-505.

Mai DH. 1995. Tertiäre Vegetationsgeschichte Europas : Methoden und Ergebnisse. Germany: Gustav Fischer Verlag.

Moore BR, Höhna S, May MR, Rannala B, Huelsenbeck JP. 2016. Critically evaluating the theory and performance of Bayesian analysis of macroevolutionary mixtures. Proceedings of the National Academy of Sciences 113.34: 9569-9574.

Morlon H, Hartig F, Robin S. 2020. Prior hypotheses or regularization allow inference of diversification histories from extant timetrees. bioRxiv: 2020.2007.2003.185074.

\section{Mosbrugger V, Favre A, Muellner - Riehl AN, Päckert M, Mulch A 2018. Cenozoic Evolution} of Geobiodiversity in the Tibeto - Himalayan Region. In: Hoorn C, Perrigo A, Antonelli A eds. Mountains, Climate and Biodiversity. UK: Wiley-Blackwell, 429-448.

Muellner-Riehl AN, Schnitzler J, Kissling WD, Mosbrugger V, Rijsdijk KF, Seijmonsbergen AC, Versteegh H, Favre A. 2019. Origins of global mountain plant biodiversity: Testing the 'mountain-geobiodiversity hypothesis'. Journal of Biogeography 46: 2826-2838.

Myers N, Mittermeier RA, Mittermeier CG, da Fonseca GAB, Kent J. 2000. Biodiversity hotspots for conservation priorities. Nature 403: 853-858. Wang Y, et al. 2018. Rapid incision of the Mekong River in the middle Miocene linked to monsoonal precipitation. Nature Geoscience 11: 944-948.

Nürk NM, Atchison GW, Hughes CE. 2019. Island woodiness underpins accelerated disparification in plant radiations. New Phytologist 224: 518-531.

Parks M, Cronn R, Liston A. 2009. Increasing phylogenetic resolution at low taxonomic levels using massively parallel sequencing of chloroplast genomes. BMC Biology 7: 84. leaves dampen temperature fluctuations in reproductive organ of an alpine Himalayan forb. Journal of Plant Ecology 8: 159-165.

Popescu SM. 2002. Repetitive changes in Early Pliocene vegetation revealed by high-resolution pollen analysis: revised cyclostratigraphy of southwestern Romania. Review of Palaeobotany and Palynology 120: 181-202.

Qu XJ, Moore MJ, Li DZ, Yi TS. 2019. PGA: a software package for rapid, accurate, and flexible batch annotation of plastomes. Plant Methods 15: 50.

761 Rabosky DL. 2014. Automatic Detection of Key Innovations, Rate Shifts, and Diversity-

762 Dependence on Phylogenetic Trees. PLOS ONE 9: e89543.

763 Rabosky DL, Goldberg EE. 2017. FiSSE: A simple nonparametric test for the effects of a binary 
character on lineage diversification rates. Evolution 71: 1432-1442.

765

766

767

768

769

770

771

772

773

774

775

776

777

778

779

780

781

782

783

784

785

786

787

788

789

790

791

792

793

794

795

796

797

798

799

800

801

802

Rabosky DL, Grundler M, Anderson C, Title P, Shi JJ, Brown JW, Huang H, Larson JG.

2014. BAMMtools: an $\mathrm{R}$ package for the analysis of evolutionary dynamics on phylogenetic trees. Methods in Ecology and Evolution 5: 701-707.

Rabosky DL, Mitchell JS, Chang J. 2017. Is BAMM Flawed? Theoretical and Practical Concerns in the Analysis of Multi-Rate Diversification Models. Systematic Biology 66: 477498.

Rambaut A, Drummond A. 2010. TreeAnnotator version 1.6. 1. University of Edinburgh, Edinburgh, UK: Available at: http://beast.bio.ed.ac.uk. [accessed 1 September 2019]

Rambaut A, Drummond AJ, Xie D, Baele G, Suchard MA. 2018. Posterior Summarization in Bayesian Phylogenetics Using Tracer 1.7. Systematic Biology 67: 901-904.

Rolland J, Salamin N. 2016. Niche width impacts vertebrate diversification. Global Ecology and Biogeography 25: 1252-1263.

Schwery O, Onstein RE, Bouchenak-Khelladi Y, Xing Y, Carter RJ, Linder HP. 2015. As old as the mountains: the radiations of the Ericaceae. New Phytologist 207: 355-367.

Sexton JP, Montiel J, Shay JE, Stephens MR, Slatyer RA. 2017. Evolution of Ecological Niche Breadth. Annual Review of Ecology, Evolution, and Systematics 48: 183-206.

Shen J, Zhang X, Landis JB, Zhang H, Deng T, Sun H, Wang H. 2020. Plastome Evolution in Dolomiaea (Asteraceae, Cardueae) Using Phylogenomic and Comparative Analyses. Frontiers in Plant Science 11: 376.

Shi Z, Raab-Straube Ev 2011. Cardueae. In: Wu ZY, Raven, P. H. \& Hong, D. Y. ed. Flora of China. Beijing \& St. Louis: Science Press \& Missouri Botanical Garden Press, 42-194.

Slatyer RA, Hirst M, Sexton JP. 2013. Niche breadth predicts geographical range size: a general ecological pattern. Ecology Letters 16: 1104-1114.

Song B, Stöcklin J, Peng D, Gao Y, Sun H. 2015. The bracts of the alpine 'glasshouse' plant Rheum alexandrae (Polygonaceae) enhance reproductive fitness of its pollinating seedconsuming mutualist. Botanical Journal of the Linnean Society 179: 349-359.

Spicer RA, Farnsworth A, Su T. 2020. Cenozoic topography, monsoons and biodiversity conservation within the Tibetan Region: An evolving story. Plant Diversity 42: 229-254.

Spicer RA, Su T, Valdes PJ, Farnsworth A, Wu F-X, Shi G, Spicer TEV, Zhou Z. 2021. Why 'the uplift of the Tibetan Plateau' is a myth? National Science Review 8: nwaa091.

Stadler T. 2011. Mammalian phylogeny reveals recent diversification rate shifts. Proceedings of the National Academy of Sciences 108: 6187.

Suchard MA, Lemey P, Baele G, Ayres DL, Drummond AJ, Rambaut A. 2018. Bayesian phylogenetic and phylodynamic data integration using BEAST 1.10. Virus Evolution 4: vey016.

Sun H, Niu Y, Chen Y-S, Song B, Liu C-Q, Peng D-L, Chen J-G, Yang Y. 2014. Survival and reproduction of plant species in the Qinghai-Tibet Plateau. Journal of Systematics and Evolution 52: 378-396. 
803 Sun H, Zhang J, Deng T, Boufford DE. 2017. Origins and evolution of plant diversity in the Hengduan Mountains, China. Plant Diversity 39: 161-166.

Sun M, Folk RA, Gitzendanner MA, Soltis PS, Chen Z, Soltis DE, Guralnick RP. 2020. Recent accelerated diversification in rosids occurred outside the tropics. Nature Communications 11: 3333.

Testo WL, Sessa E, Barrington DS. 2019. The rise of the Andes promoted rapid diversification in Neotropical Phlegmariurus (Lycopodiaceae). New Phytologist 222: 604-613.

Tsukaya H. 2002. Optical and anatomical characteristics of bracts from the Chinese "glasshouse" plant, Rheum alexandrae Batalin (Polygonaceae), in Yunnan, China. Journal of Plant Research 115: 59-63.

Vrba ES. 1987. Ecology in relation to speciation rates: some case histories of Miocene-Recent mammal clades. Evolutionary Ecology 1: 283-300.

Wang YJ, Susanna A, Von Raab-Straube E, Milne R, Liu JQ. 2009. Island-like radiation of Saussurea (Asteraceae: Cardueae) triggered by uplifts of the Qinghai-Tibetan Plateau. Biological Journal of the Linnean Society 97: 893-903.

Warren DL, Glor RE, Turelli M. 2010. ENMTools: a toolbox for comparative studies of environmental niche models. Ecography 33: 607-611.

Wen J, Zhang J, Nie Z-L, Zhong Y, Sun H. 2014. Evolutionary diversifications of plants on the Qinghai-Tibetan Plateau. Frontiers in Genetics 5: 4.

Whitfield JB, Lockhart PJ. 2007. Deciphering ancient rapid radiations. Trends in Ecology \& Evolution 22: 258-265.

Wicke S, Schneeweiss GM, dePamphilis CW, Muller KF, Quandt D. 2011. The evolution of the plastid chromosome in land plants: gene content, gene order, gene function. Plant Molecular Biology 76: 273-297.

Xing Y, Ree RH. 2017. Uplift-driven diversification in the Hengduan Mountains, a temperate biodiversity hotspot. Proceedings of the National Academy of Sciences: 201616063.

Xu L-S, Herrando-Moraira S, Susanna A, Galbany-Casals M, Chen Y-S. 2019. Phylogeny, origin and dispersal of Saussurea (Asteraceae) based on chloroplast genome data. Molecular Phylogenetics and Evolution 141: 106613.

838 Yang Y, Chen J, Song B, Niu Y, Peng D, Zhang J, Deng T, Luo D, Ma X, Zhou Z. 2019.

839 Advances in the studies of plant diversity and ecological adaptation in the subnival ecosystem 840 of the Qinghai-Tibet Plateau. Chinese Science Bulletin 64: 2856-2864.

841 Yang Y, Sun H. 2009. The Bracts of Saussurea velutina (Asteraceae) Protect Inflorescences from 
842 Fluctuating Weather at High Elevations of the Hengduan Mountains, Southwestern China.

843 Arctic, Antarctic, and Alpine Research 41: 515-521.

844 Zachos JC, Dickens GR, Zeebe RE. 2008. An early Cenozoic perspective on greenhouse

$845 \quad$ warming and carbon-cycle dynamics. Nature 451: 279-283.

846 Zhang J-Q, Meng S-Y, Allen GA, Wen J, Rao G-Y. 2014. Rapid radiation and dispersal out of

847 the Qinghai-Tibetan Plateau of an alpine plant lineage Rhodiola (Crassulaceae). Molecular

$848 \quad$ Phylogenetics and Evolution 77: 147-158.

849 Zhang X, Deng T, Moore MJ, Ji Y, Lin N, Zhang H, Meng A, Wang H, Sun Y, Sun H. 2019a.

850 Plastome phylogenomics of Saussurea (Asteraceae: Cardueae). BMC Plant Biology 19: 290.

851 Zhang X, Sun Y, Landis JB, Lv Z, Shen J, Zhang H, Lin N, Li L, Sun J, Deng T, et al. 2020.

852 Plastome phylogenomic study of Gentianeae (Gentianaceae): widespread gene tree

853 discordance and its association with evolutionary rate heterogeneity of plastid genes. $B M C$

$854 \quad$ Plant Biology 20: 340.

855 Zhang Y, Tang R, Huang X, Sun W, Ma X, Sun H. 2019b. Saussurea balangshanensis sp. nov.

856 (Asteraceae), from the Hengduan Mountains region, SW China. Nordic Journal of Botany 37:

$857 \quad$ https://doi.org/10.1111/njb.02078. 
bioRxiv preprint doi: https://doi.org/10.1101/2021.03.15.435394; this version posted March 16, 2021. The copyright holder for this preprint

(which was not certified by peer review) is the author/funder, who has granted bioRxiv a license to display the preprint in perpetuity. It is made available under aCC-BY-NC-ND 4.0 International license.

\section{Figure legends:}

859 Fig. 1 Diversification dynamics of Saussurea inferred from BAMM analysis. (a)

860 BAMM identified two shifts in diversification rates (represented by arrows). The time

861 of three clades beginning to diversify is provided. (b) Rates-through-time plots of all

862 Saussurea species and three main clades separately, with trends in global climate

863 change over 12 million years (Zachos et al. 2008) depicted. (c-d) BAMM tip rates of

864 three clades and four morphology-based subgenera of Saussurea, respectively.

865 Fig. 2 Paleoenvironment-dependent diversification processes in Saussurea. The best-

866 fit paleoenvironment-dependent model implemented in RPANDA shows negative

867 dependence between paleotemperatures (a) and speciation rate (b).

868 Fig. 3 Binary trait dependent diversification of Saussurea inferred from HiSSE

869 analysis. Speciation, extinction and net diversification rates are calculated by the

870 model-averaged marginal ancestral state reconstruction for four binary traits: (a)

871 stemless (0) vs. cauliferous (1), (b) stem glabrous (0) vs. densely haired (1), (c) the

872 absence (0) vs. presence (1) of leafy bracts, and (d) capitula solitary (0) vs. numerous 873 (1).

874 Fig. 4 Multistate trait dependent diversification of Saussurea estimated from MuSSE 875 analysis. Marginal distributions of net diversification rates are estimated by the

876 MCMC run of 5, 000 generations for four multistate traits: (a) leaves margin entire (1)

877 vs. pinnately lobed (2) vs. both types (3), (b) leaves glabrous (1) vs. sparsely haired

878 (2) vs. densely haired (3), (c) phyllary in <5 (1) vs. 5 (2) vs. 6 (3) vs. $>6$ (4) rows, and

879 (d) phyllary glabrous (1) vs. sparsely haired (2) vs. densely haired (3) vs. appendage 880 (4).

881 Fig. 5 Speciation rates of Saussurea correlated with ecological factors based on the 882 QuaSSE best-fitted model and ES-sim tests. Both (a) niche breadth and (b) species 883 range size (log-transformed) show positive sigmoidal curves in QuaSSE analysis with 884 the midpoints (represented by the red dashed line) of 0.729 and 11.433 on the $\mathrm{x}$-axis 885 respectively. EM-sim tests show significant positive relationships between DR 886 speciation rates and (c) niche breadth and (d) species range size. Species from three 887 clades are in different colors. 
bioRxiv preprint doi: https://doi.org/10.1101/2021.03.15.435394; this version posted March 16, 2021. The copyright holder for this preprint (which was not certified by peer review) is the author/funder, who has granted bioRxiv a license to display the preprint in perpetuity. It is made 889 Table 1 Results of RPANDA analyses.

\begin{tabular}{|c|c|c|c|c|c|c|c|}
\hline Models & $\mathbf{N P}$ & $\log \mathrm{L}$ & AICc & $\lambda_{0}$ & $\alpha$ & $\mu_{0}$ & $\boldsymbol{\beta}$ \\
\hline Constant birth-death (1) & 2 & -325.7908 & 655.6424 & 0.7214 & NA & 0.3714 & NA \\
\hline$\lambda_{\text {Time }}$ and $\mu_{\text {constant }}(2)$ & 3 & -325.2570 & 656.6364 & 0.6801 & -0.0618 & 0.1562 & NA \\
\hline$\lambda_{\text {Temp. and }} \mu_{\text {constant }}$ (3) & 3 & -324.4240 & 654.9705 & 0.7585 & -0.0933 & 0.1610 & $\mathbf{N A}$ \\
\hline$\lambda_{\text {constant }}$ and $\mu_{\text {Time }}(4)$ & 3 & -325.4236 & 656.9698 & 0.7020 & NA & 0.3051 & 0.0475 \\
\hline$\lambda_{\text {constant }}$ and $\mu_{\text {Temp. }}$ (5) & 3 & -325.4355 & 656.9930 & 0.649 & NA & 0.1445 & 0.2067 \\
\hline$\lambda_{\text {Time }}$ and $\mu_{\text {Time }}(6)$ & 4 & -325.1840 & 658.5732 & 0.6840 & -0.0460 & 0.1843 & -0.0036 \\
\hline$\lambda_{\text {Temp. and }} \mu_{\text {Temp. }}$ (7) & 4 & -323.9815 & 656.168 & 0.693 & -0.0017 & 0.1910 & 0.1159 \\
\hline
\end{tabular}

890 Bold columns represent the best model, in which speciation rate is negative dependence $(\alpha<0)$ to

891 past temperature and extinction rate is constant. Detailed model sets are described in Condamine

892 et al. (2013). Abbreviations: NP, number of parameters; logL, log-likelihood; AICc, corrected

893 Akaike Information Criterion. Parameter estimates: $\lambda_{0}$ and $\mu_{0}$, speciation and extinction rates for a

894 given environmental variable; and $\alpha, \beta$, parameter controlling variation of speciation and

895 extinction with paleo-environment, respectively.

896 
bioRxiv preprint doi: https://doi.org/10.1101/2021.03.15.435394; this version posted March 16, 2021. The copyright holder for this preprint (which was not certified by peer review) is the author/funder, who has granted bioRxiv a license to display the preprint in perpetuity. It is made 897 Table 2 Summary of the mean rate values for four binary traits in HiSSE and FiSSE

898 analysis.

\begin{tabular}{|c|c|c|c|c|c|}
\hline Trait & $\begin{array}{l}\text { HiSSE } \\
\lambda\end{array}$ & $\mu$ & $\lambda-\mu$ & $\begin{array}{l}\text { FiSSE } \\
\lambda\end{array}$ & $p$-value \\
\hline Stemless & 0.5947 & 0.2415 & 0.3532 & 0.9011 & \\
\hline Cauliferous & 1.0893 & 0.3821 & 0.4502 & 0.9264 & 0.4416 \\
\hline Glabrous & 1.7230 & 1.2100 & 0.5128 & 0.8971 & \\
\hline Densely haired & 0.5531 & 0.2223 & 0.3308 & 0.8674 & 0.4096 \\
\hline Normal & 0.8925 & 0.6849 & 0.2076 & 0.8987 & \\
\hline Bracts & 1.3951 & 0.8752 & 0.5199 & 0.9412 & 0.5614 \\
\hline Capitula solitary & 1.6920 & 0.9969 & 0.6952 & 1.0825 & \\
\hline Capitula numerous & 0.5661 & 0.5253 & 0.0408 & 0.7828 & 0.0240 \\
\hline
\end{tabular}

899 Traits with higher net diversification rates $(\lambda-\mu)$ are in bold. For HiSSE analysis, mean rate values

900 are calculated from the model-averaged marginal ancestral state reconstruction, and detailed

901 model tests are provided in Supporting Information Table S7. For FiSSE analysis, the significant

$902 p$-values for adjusted results are in bold.

903

904 Table 3 Summary of ES-sim tests for correlation between speciation rate and

905 continuous ecological factors.

\begin{tabular}{|c|c|c|c|c|}
\hline \multirow{2}{*}{$\begin{array}{l}\text { Ecological } \\
\text { factors }\end{array}$} & \multicolumn{2}{|c|}{ ES-sim (DR statistic) } & \multicolumn{2}{|c|}{$\begin{array}{l}\text { ES-sim (Inverse equal } \\
\text { splits statistic) }\end{array}$} \\
\hline & $\rho$ & $p$-value & $\rho$ & $p$-value \\
\hline ClimatePC1 & 0.170 & 0.359 & 0.188 & 0.335 \\
\hline ClimatePC2 & 0.098 & 0.649 & 0.095 & 0.635 \\
\hline Niche breadth & 0.363 & 0.027 & 0.387 & 0.019 \\
\hline Range size & 0.399 & 0.018 & 0.411 & 0.011 \\
\hline
\end{tabular}

906 The significant $p$-values for the correlation are in bold. Rho $(\rho)$ is the Pearson's correlation

907 coefficient. Both the DR statistic and the default inverse equal splits statistic were used as reliable

908 estimators. Detailed test statistic described in Harvey \& Rabosky (2018).

909 
(a)

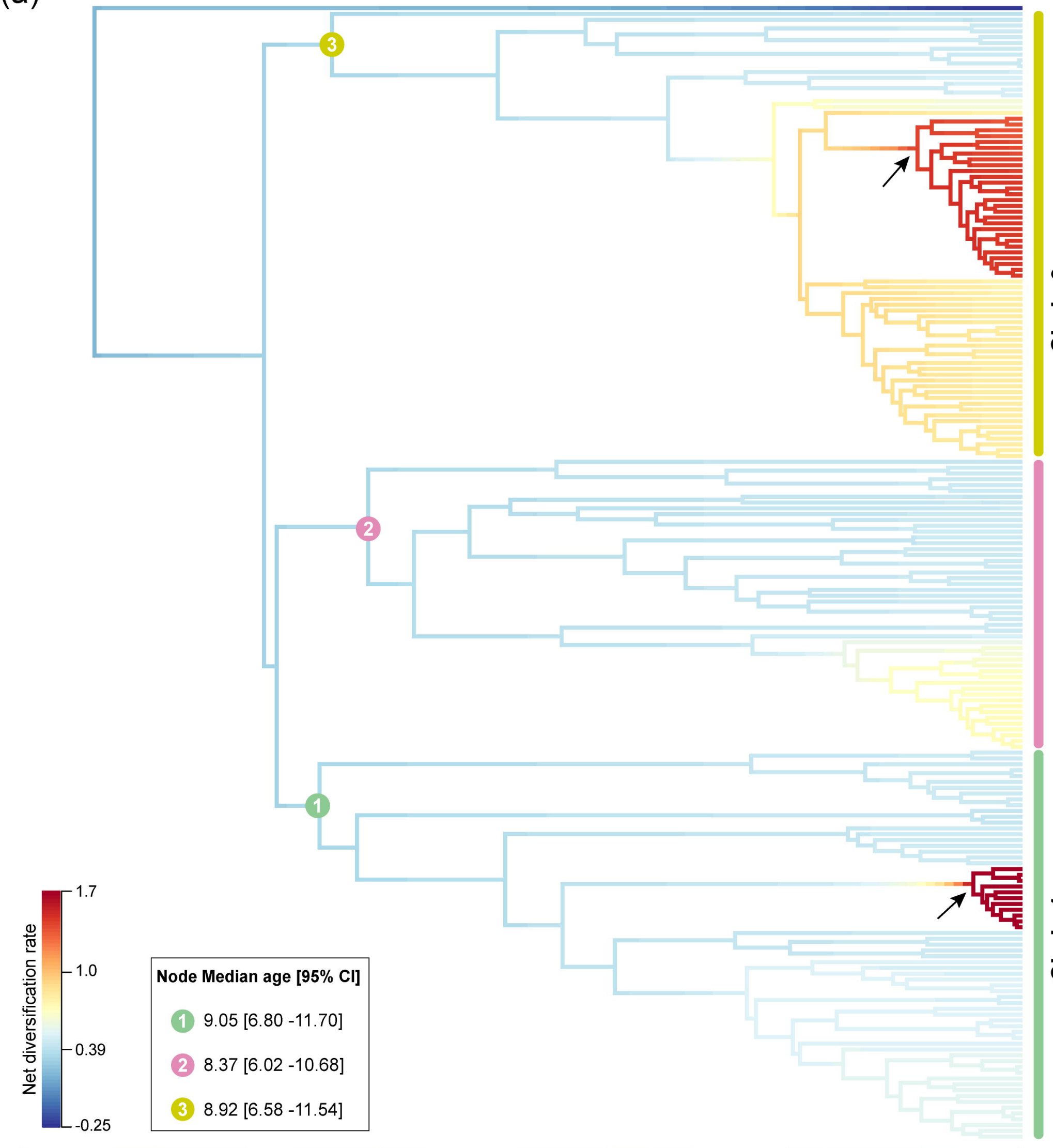

(b)
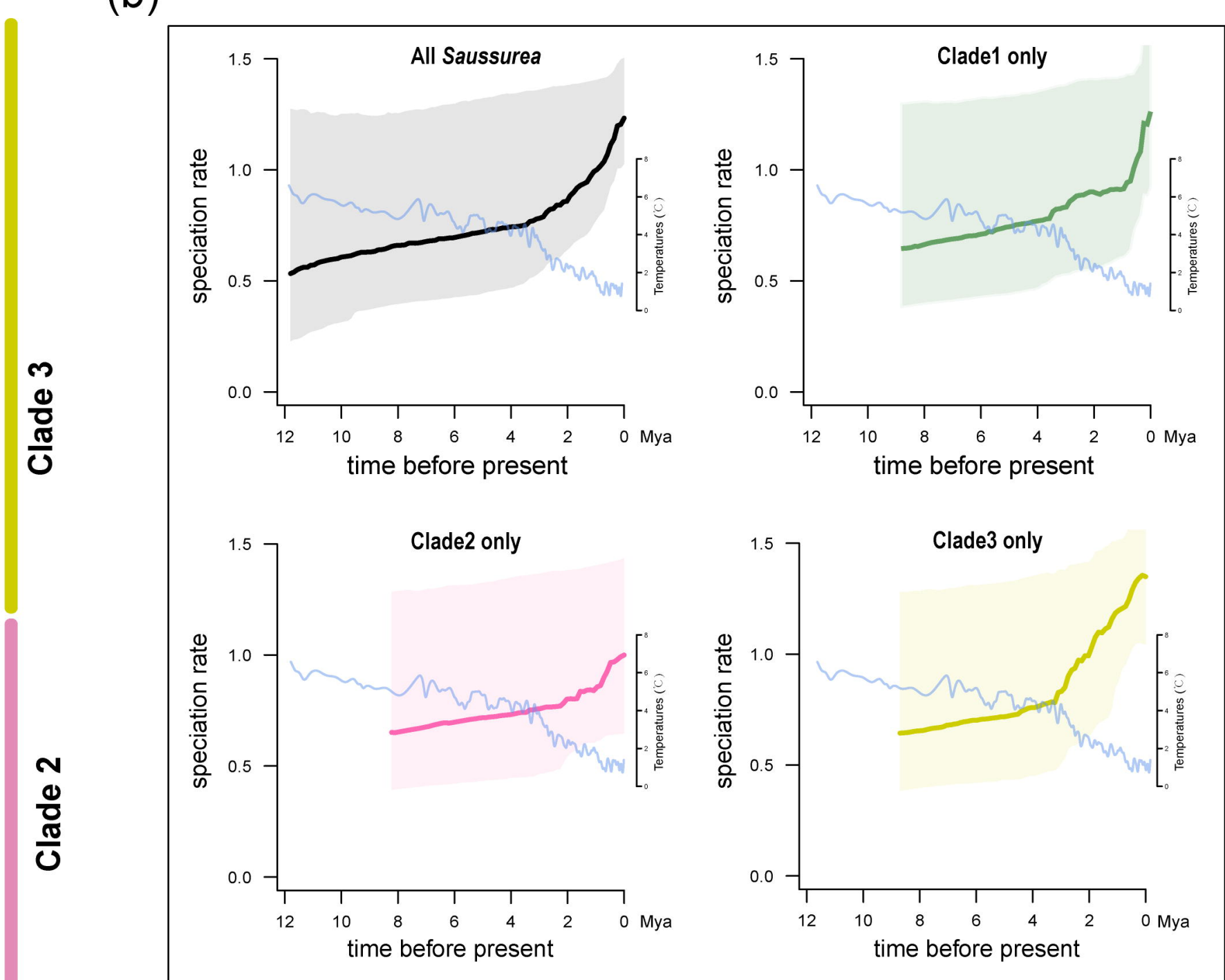

(c)

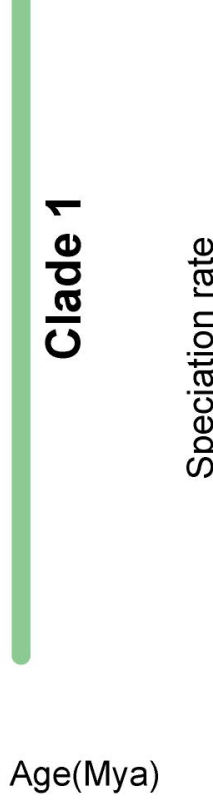

(d)

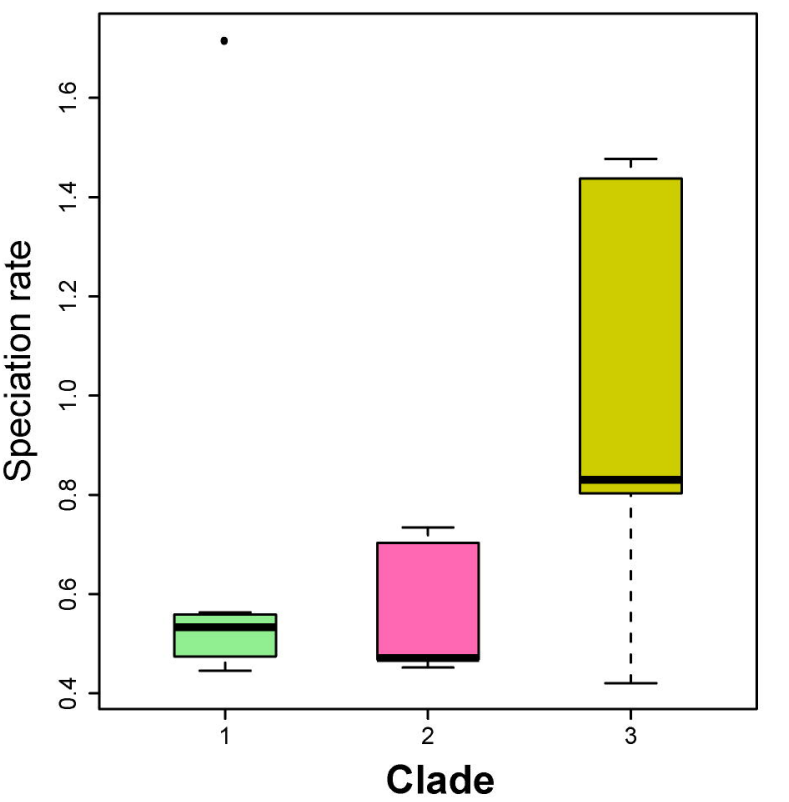

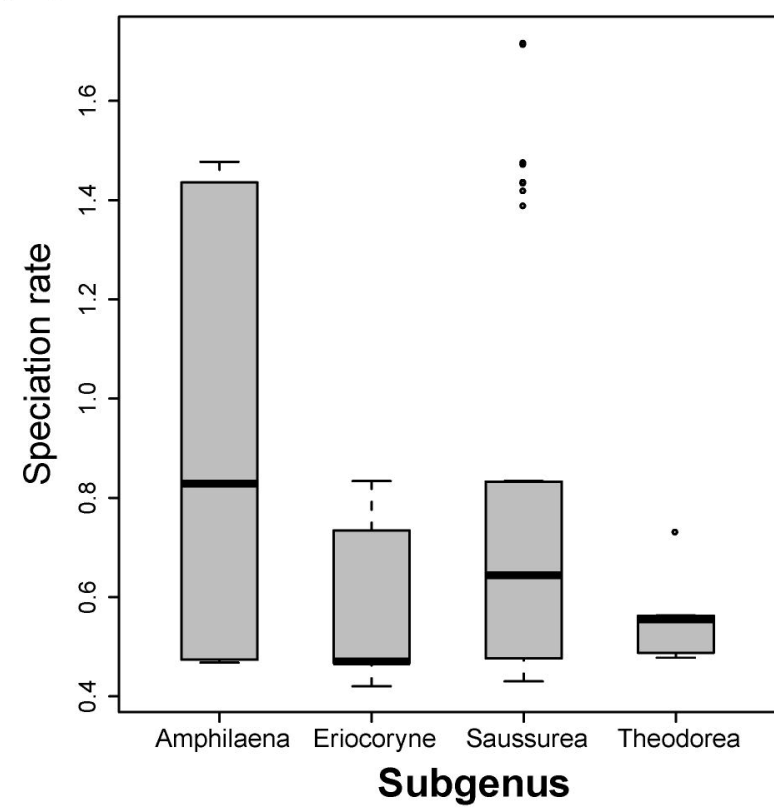


(a) Global paleo-temperatures over the last 12 Myrs

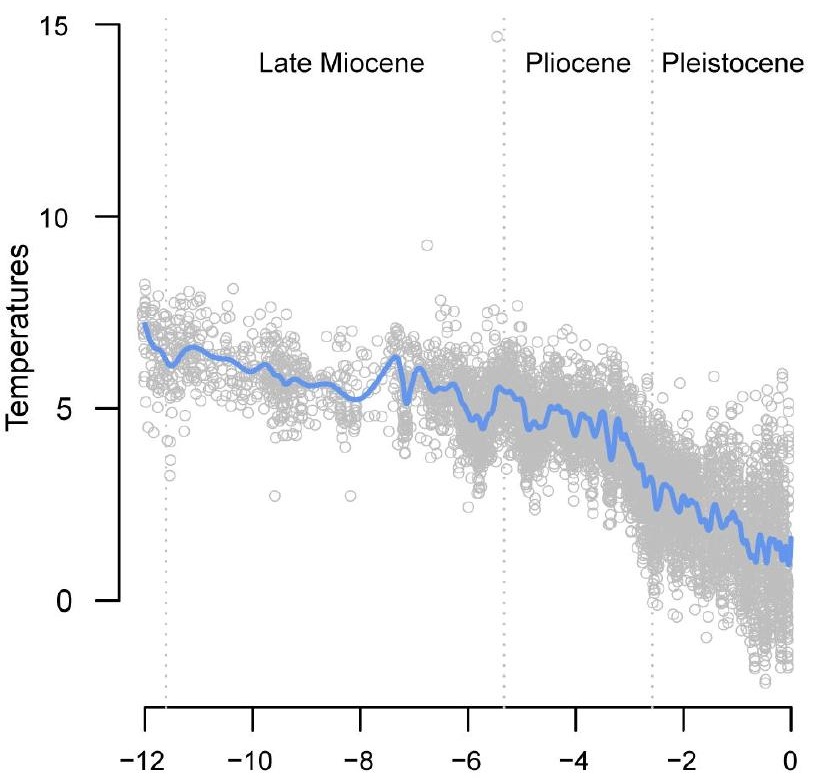

(b) Speciation is negatively correlated to past temperatures

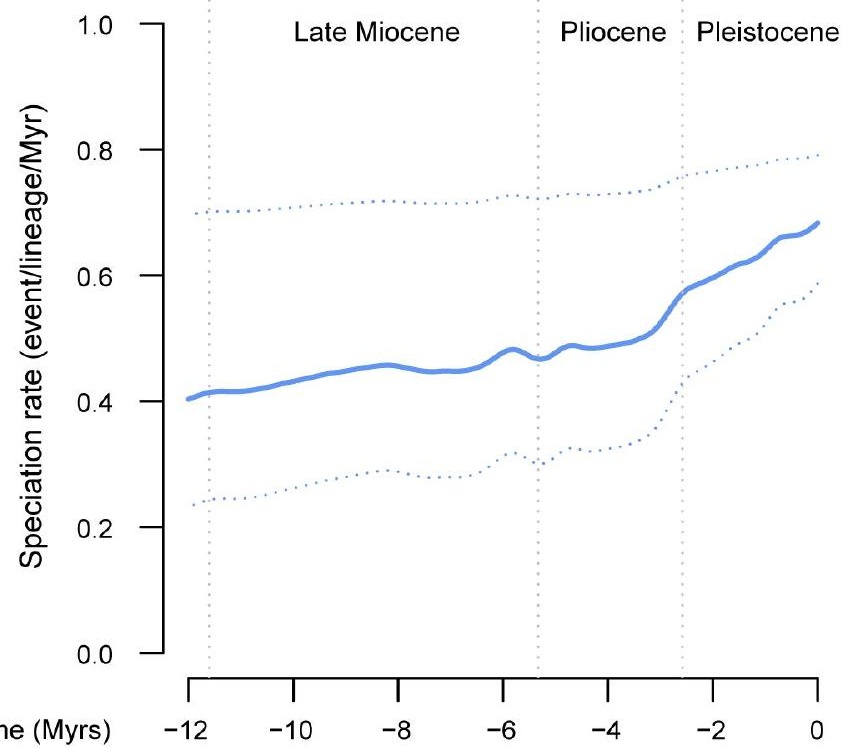


(a)

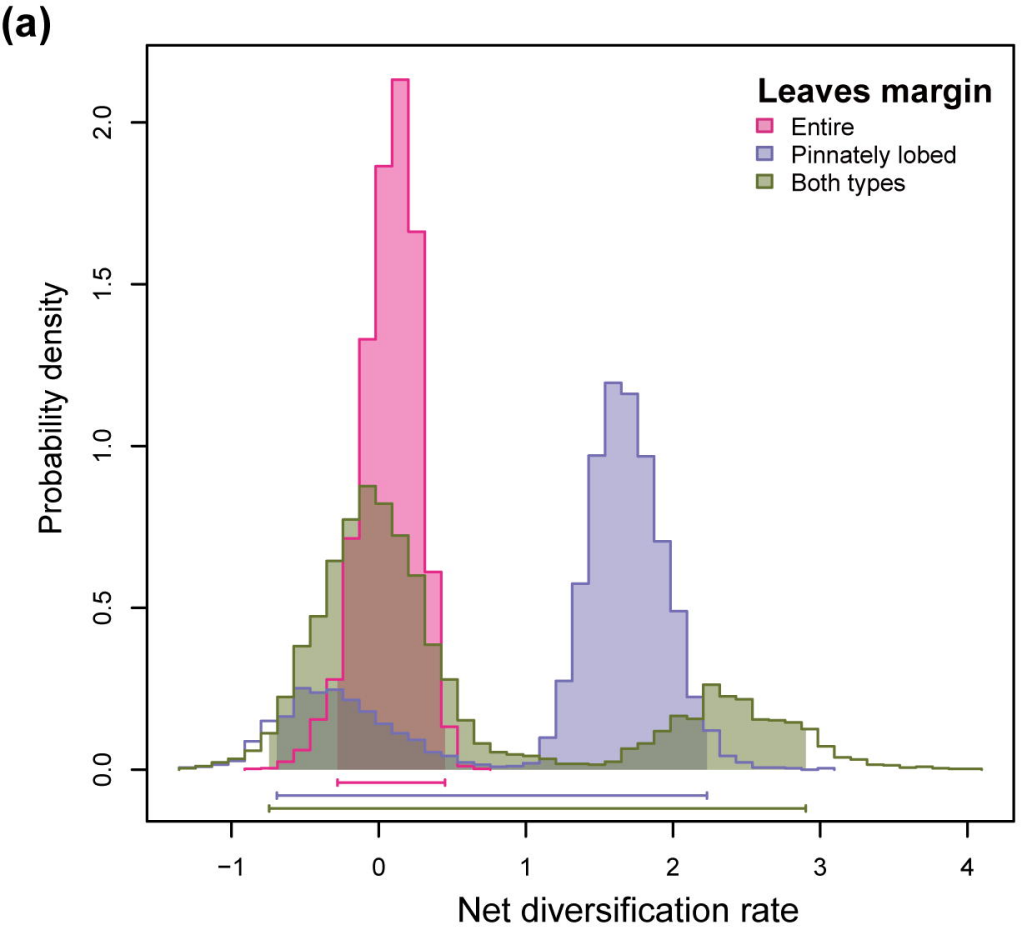

(c)

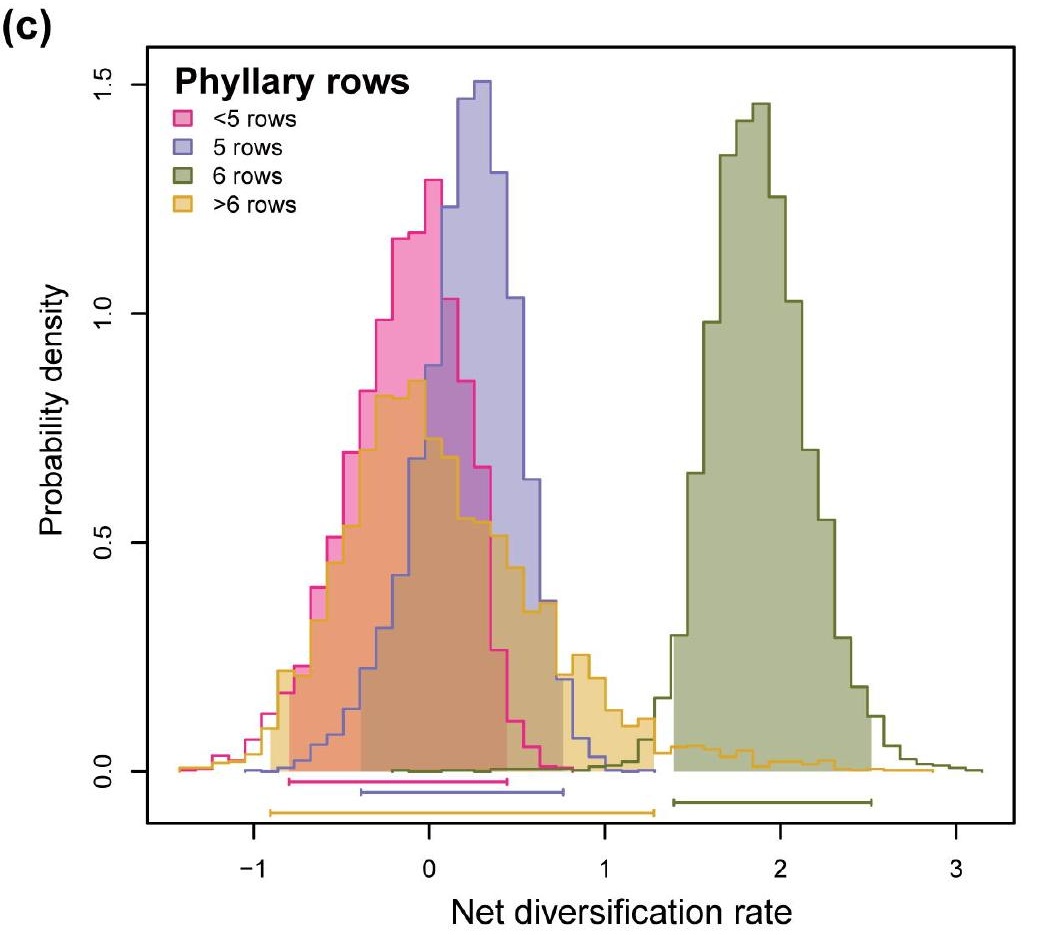

Net diversification rate
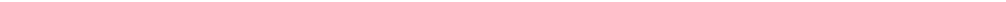

(d)

(b)

(


(b)
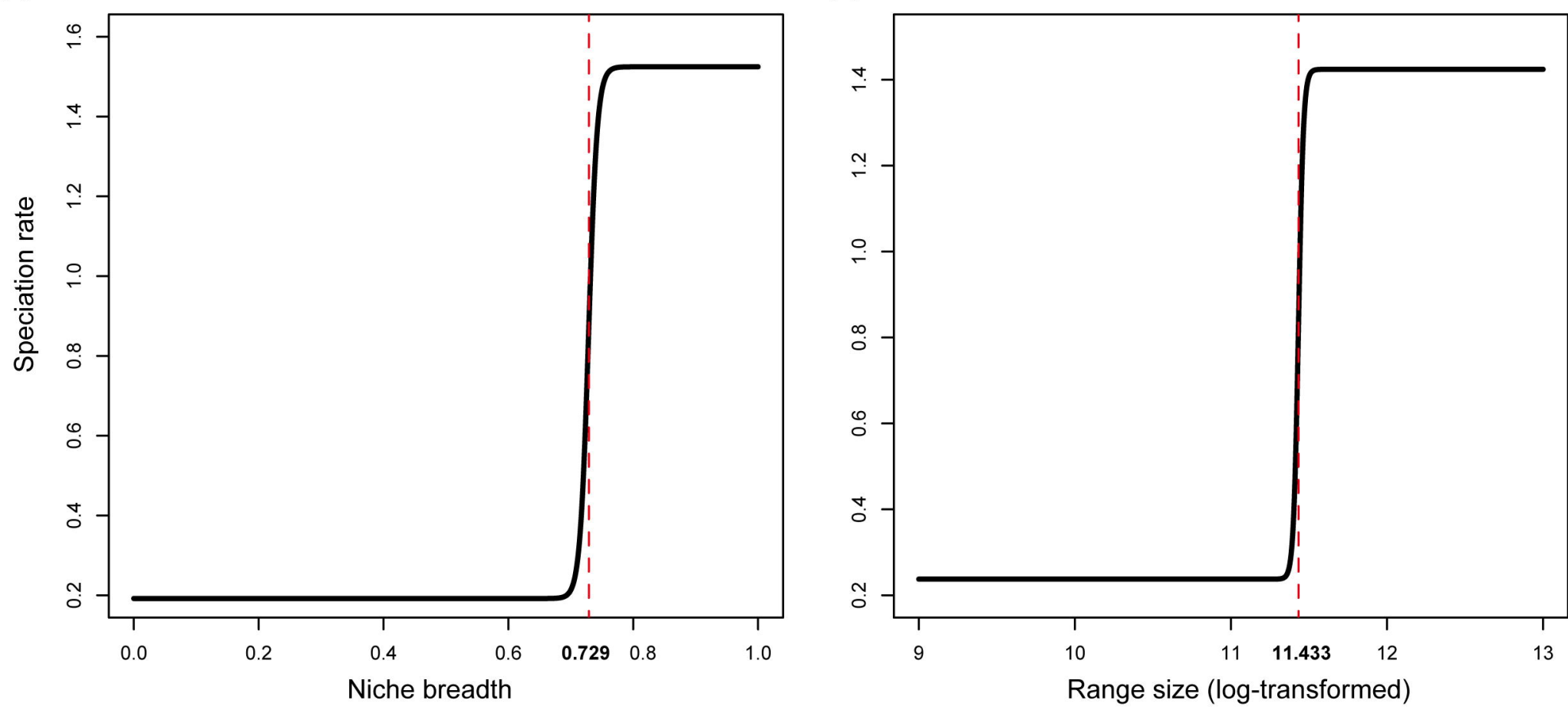

(c)

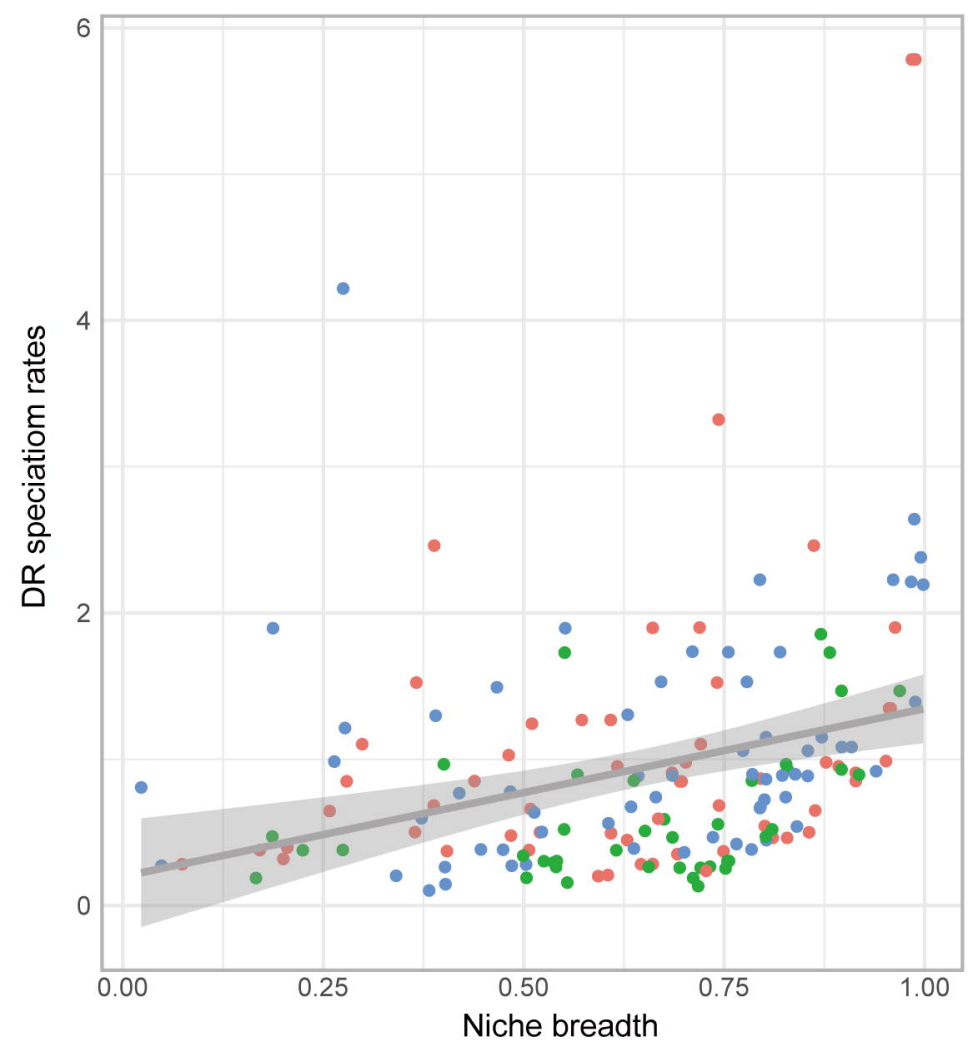

(d)

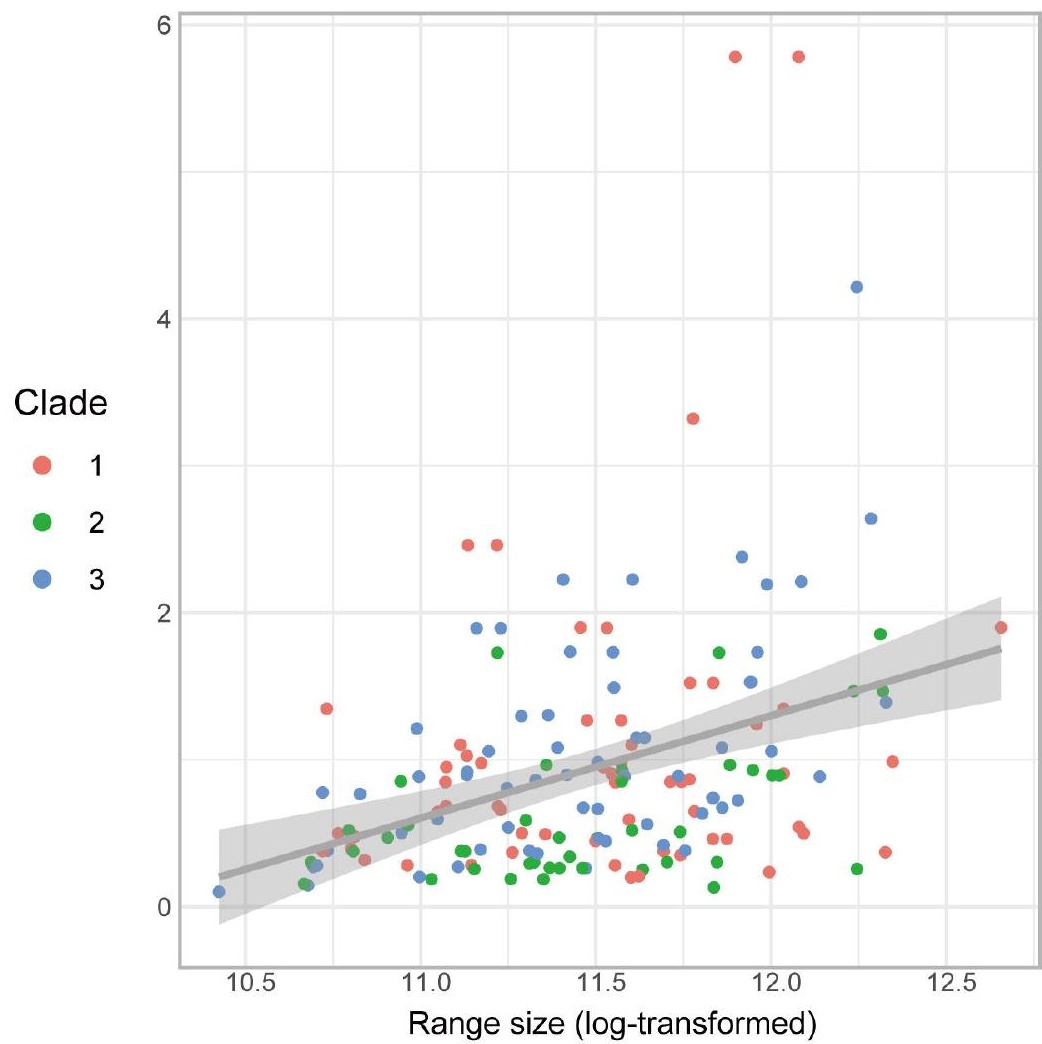

